\title{
Two-Step Method for Millimeter-Wave Antenna Performance Assessment in 5G Smartphones
}

\author{
Filipa S. S. Fernandes $\left(\mathbb{D},{ }^{1}\right.$ Christian Rom $\mathbb{D}^{1},{ }^{2}$ António Rodrigues $\left(\mathbb{D},{ }^{3}\right.$ Simon Svendsen $(\mathbb{D}){ }^{2}$ \\ and Ole Jagielski (iD ${ }^{4}$ \\ ${ }^{1}$ Instituto Superior Técnico, Lisbon 1049-001, Portugal \\ ${ }^{2}$ Nokia Bell Labs, Aalborg 9220, Denmark \\ ${ }^{3}$ Department of Electrical and Computer Engineering, IST-University of Lisbon and Instituto de Telecomunicações, \\ Lisbon 1049-001, Portugal \\ ${ }^{4}$ Intel Mobile Communications, Aalborg 9220, Denmark \\ Correspondence should be addressed to Filipa S. S. Fernandes; filipa.fernandes@ist.utl.pt
}

Received 25 November 2020; Revised 5 May 2021; Accepted 27 May 2021; Published 11 June 2021

Academic Editor: Mourad Nedil

Copyright @ 2021 Filipa S. S. Fernandes et al. This is an open access article distributed under the Creative Commons Attribution License, which permits unrestricted use, distribution, and reproduction in any medium, provided the original work is properly cited.

\begin{abstract}
A critical challenge for $5 \mathrm{G}$ is transitioning to the $\mathrm{mm}$-Wave spectrum. Despite providing unprecedented data rates, $\mathrm{mm}$-Waves also suffer high path loss, atmospheric absorption, and higher fluctuating channel conditions, sparking numerous paradigm shifts in the smartphone industry. Extending $\mathrm{mm}$-Wave communications to smartphones requires first a comprehensive study to identify the antenna design/smartphone implementation challenges that impact the quality of communications. This work proposes a two-step assessment metric, the mmWAESI, to evaluate $\mathrm{mm}$-Wave antennas' potential and limitations regarding their impact on system performance. First, it analyzes the spatial distribution of the smartphone-integrated beam steering array's radiated power. Then, it evaluates the antenna's influence on the MIMO performance, using a discrete, time-variant geometrical MIMO channel simulator to recreate any mm-Wave propagation scenario. For enhanced accuracy, mmWAESI accounts simultaneously for several communication aspects: antenna type, realistic radiation patterns, mobile phone form factor constraints, phone orientation, and user influence. The method is illustrated for two different 4-element linear arrays at $39 \mathrm{GHz}$, based on patch or monopole elements, integrated into smartphones. Their performance is compared under similar conditions, revealing that, unless array switching is employed, the smartphone's form factor and user influence will mask any potential advantage of the unperturbed array characteristics.
\end{abstract}

\section{Introduction}

$5 \mathrm{G}$ is the wireless technology being currently developed to sustain the high amounts of data rate, connections, bandwidth and low latency requirements that come with more users, devices, and ambitious endeavors such as Smart Cities or Autonomous Vehicles $[1,2]$. However, the topic of $5 \mathrm{G}$ is still somewhat abstract when it comes to millimeter-wave (mm-Wave) antenna technologies. In the $\mathrm{mm}$-Wave domain, contrary to sub- $6 \mathrm{GHz}$ antenna design for cellular hand held devices, the antennas take a much bigger part in the performance and feasibility of the system and should not be disregarded [3]. 5G mm-Wave raises a major antenna paradigm shift, since it requires higher gain and beam steering ability [4]. Therefore, this new dynamic antenna behavior calls for a weaving of beam control and MultipleIn-Multiple-Out (MIMO) functions.

Despite the existence of $\mathrm{mm}$-Wave antennas in radio infrastructures being deployed in the near future, $\mathrm{mm}$-Wave antenna technologies for $5 \mathrm{G}$ cellular handsets are still at their early stages. There is still no standardized method to design and implement these antennas in mobile phones due to the lack of knowledge on $\mathrm{mm}$-Wave $5 \mathrm{G}$ wireless system benchmarks [5]. This prevents the impact evaluation of 
design parameters such as user influence, handset effects, and gain coverage, since there is not a reference for comparison.

This paper proposes a methodology, the mm-Wave Antenna Evaluation for Smartphone Implementation (mmWAESI) method, a practical procedure intended to evaluate the performance of different MIMO mm-Wave antenna implementations in a $5 \mathrm{G}$ system for smartphone implementation. It does so by blending two dimensions of antenna performance analysis: multipath channel modeling and realistic antenna characterization and environment constraints. Despite being equally important to the feasibility of antenna array implementation in $\mathrm{mm}$-Wave communications, these two approaches are usually studied separately. Therefore, the mmWAESI combines them through a two-step metric that incorporates the real effects of $\mathrm{mm}$-Wave antenna design constraints in $5 \mathrm{G}$ system performance evaluation. In the first step of the method, the Coverage Study Step, the array's beamforming potential is evaluated in terms of spatial gain coverage using a MATLAB developed toolbox, the Antenna Pattern Analysis Toolbox (APA TBX), to create a coverage area percentage curve. In the second step of the method, the MIMO Performance Step, a MATLAB developed channel model was implemented to create different types of mm-Wave propagation scenarios and measure how contingent the MIMO performance is on the user rotating the user equipment (UE), disturbing its alignment with the base station (BS). This channel model employs antenna radiation patterns and characteristics obtained from the 3D electromagnetic solver Computer Simulation Technology (CST) Microwave Studio, which depict the antennas behavior more realistically than purely mathematical-based models usually used for channel simulations. Moreover, it accounts for the beam steering and codebook size, the effects of the handset's metallic casing, the phone's rotation and orientation with reference to the base station, the user blockage, and the depolarization effects, and it also includes configurable multipath scenarios.

The mmWAESI method can be used to choose between antennas types for a particular smartphone implementation scenario. The antennas would go through the two steps, Coverage Study Step and MIMO Performance Step, under the same testing conditions so that their swept area coverage potential and MIMO channel performance could be compared. It can also be used to test different array configurations, their positioning in the smartphone, and types of hand grips in variable environment conditions, facilitating the smartphone $\mathrm{mm}$-Wave antenna design and prototyping.

Although this study could also be accomplished through over-the-air (OTA) testing, it would be a much more expensive, complex, and time consuming process. Therefore, it is crucial to first pursue the development of realistic models, such as mmWAESI, to predict the UE's behavior when facing the challenges of $\mathrm{mm}$-Wave propagation.

The proposed method is illustrated in this paper using two promising mm-Wave antenna array families: a Patch Based Solution (PBS) and a Monopole Based Solution (MBS). These antennas were implemented in the bottom of the smartphone as a single linear 4-element uniform array, for simplicity, and compared under three different scenarios:
Line-of-Sight scenario (LOS): this is a reference scenario where there is solely the unobstructed direct path without any source of multiple paths.

Line-of-Sight with multipath scenario (LOS + multipath): scatterers are randomly positioned in the vicinity of the UE and the BS to create a multipath rich MIMO channel.

No-Line-of-Sight with multipath scenario (NLOS + multipath): for this scenario, the LOS component is expected to be almost null, due to the obstruction of the LOS path between the UE and the BS by an obstacle; however, adding multiple paths to the environment is expected to give the signal alternative paths to the one that is blocked so that communications can take place.

The remainder of this paper is organized as follows: Section 2 summarizes the challenges identified in the literature regarding the implementation of $\mathrm{mm}$-Wave antennas in a smartphone. Section 3 describes the reasoning behind the selection of the two mm-Wave antenna types used for the illustrative study, as well as the details of their design. Section 4 analyzes the impact of antenna patternaltering factors mentioned in Section 2, namely, the smartphone's structure and user influence. The two steps of the mmWAESI method, Coverage Study Step and MIMO Performance Step, are described in Sections 5 and 6, respectively. Finally, Section 7 presents the results of the illustrative study comparing the PBS array with the MBS array in terms of smartphone implementation feasibility and performance.

\section{Challenges on mm-Wave Antenna Design for Smartphone Implementation}

In this section, some of the major challenges for mm-Wave antenna design and smartphone implementation are presented, as well as the proposed approaches in the literature to mitigate them.

The first obstacle is the mm-Wave's poor propagation conditions. Despite offering a wider bandwidth, which is very appealing for large data rate communications [6], there is strong path loss, atmospheric absorption, low penetration through objects, and little diffraction around obstacles [7, 8]. To counteract this, increased array gain is required to compensate for the huge losses. This is only viable because $\mathrm{mm}$-Wave antennas are electrically small and fit better into tighter spaces such as the mobile phone. In [9], a pair of sixteen-element patch arrays is placed on the top and bottom of the phone, whereas [10] presents eight modules of eightelement patch arrays distributed in the handset.

It is known that antenna arrays enable beam steering. Using this, the regular MIMO approach adopted for mobile phones can be redesigned to combine MIMO's Spatial Multiplexing with beamforming [11, 12]. In [10], the beamforming modules are meant to transmit 8 separate data streams, but if any of the modules is blocked, the rest can either continue to function in a MIMO Spatial Multiplexing mode (with one less array) or simply work independently.

However, accommodating for a large number of antennas in a mobile phone is challenging. Nowadays, there is a 
lack of space for hardware in the handset [3]. These compact form factors, besides having to accommodate for other wireless technologies, need to integrate batteries, large screens, multiple cameras, fingerprint scanning, gyroscope, vibrator, and more to ensure the best user experience. This resulted in a lot of articles for compact and creative antenna solutions to optimize the space usage within the phone. In [11], a dual polarized antenna is used: two arrays are implemented in a phone with switchable polarization, halving the number of arrays needed while doubling the spectral efficiency.

Another big challenge for the antenna design is that most state-of-the-art smartphones also have metallic casings. These serve an aesthetic purpose, while providing structural sturdiness and heat dissipation [10, 13]. However, these design choices place another hurdle for $\mathrm{mm}$-Wave antenna placement, since bigger screens and metallic form factors increase the amount of metal in the antenna's proximity. For these high frequencies, metal is responsible for a dramatic radiation pattern loss of shape. Therefore, it is vital to include the form factor in the antenna behavior studies.

Besides these design constraints, the user's influence in the antenna behavior must be considered. Firstly, the user's body, when in proximity to antennas at these frequencies, blocks any signal that comes in its direction [14]. In [15], tests were made at $15 \mathrm{GHz}$ to show that the body is a clear obstacle to signal propagation. For $28 \mathrm{GHz}$, in [16], results show that the user blockage effect is even more severe for higher frequencies. A hand or head close to the phone produces major disturbances in the antenna's radiation pattern and must be accounted for in test simulations. Secondly, it is hard to predict how the user will orient the handset in relation to the BS. This can interfere with the polarization alignment between the UE and the BS, deteriorating the MIMO performance. Ultimately, this could make solutions such as the dual polarized antennas much less effective than initially idealized.

The communication channel takes a big part in determining the MIMO system performance. Therefore, it is wise to establish an accurate and reliable channel model that recreates the environment and propagation features of $\mathrm{mm}$-Wave, while incorporating all the aforementioned antenna design parameters [17]. However, since this is a novel subject still under investigation, there are not too many articles in the literature that rigorously account for all these design and implementation requirements simultaneously. Some of these models already exist, such as [18], but resort to simplifications regarding antenna modeling and fail to take some of the discussed design parameters into consideration (the smartphone chassis or the user influence). Moreover, some of the $\mathrm{mm}$-Wave antenna proposals for smartphone integration can present overly optimistic performance results by disregarding less favorable propagation conditions scenarios. In [5], the measurements related to user blockage, one of the most significant challenges of $\mathrm{mm}$-Wave propagation, are only conducted for the scenario where the UE and the BS are facing each other, which is the optimal positioning to maximize throughput performance.
To tackle all these challenges, this paper proposes the mmWAESI method that provides a more realistic analysis in terms of coverage and MIMO performance for mm-Wave antenna implementation in smartphones.

\section{3. mm-Wave Antenna Types for Smartphone Implementation}

3.1. Selection Criteria. PBS is a very popular antenna type in the literature. The low profile and high gain values when used in an array configuration suggest that the PBS is a good candidate for 5G smartphone implementation, not to mention its dual polarization ability.

However, the ideal narrow beams obtained with the PBS in free space might lose their shape when realistic environment conditions come into play (such as the phone chassis or the user's influence). If realistic radiation pattern prediction is not a reliable option, then basing an antenna's design on achieving a specific beam behavior might result in a vain effort.

Instead of focusing solely on gain, it is imperative to also analyze swept coverage. A good option to improve this aspect would be an antenna array element with a broader radiation pattern, such as the MBS. These antennas have higher bandwidth; are also smaller, so they would not interfere too much with legacy antennas in the handset; and are less complex to implement.

Therefore, these are the two candidate antenna solutions chosen for the illustrative assessment of the mmWAESI method. The goal is to be able to identify which one is best suited for smartphone implementation based on swept coverage area and MIMO channel performance, while considering all the design and implementation restrictions enumerated above.

3.2. Antenna Array Design. The antenna solutions were designed as linear 4-element arrays in CST Microwave Studio [19]. They were tuned for $39 \mathrm{GHz}$. In this project, the impedance match was ensured through manipulation of the antenna's electrical parameters, since for such high frequencies it is not feasible to produce the required discrete circuitry elements. The number of antenna elements for the linear array was chosen as a compromise between gain and coverage, so that the beams had enough gain to cover the propagation losses induced by $\mathrm{mm}$-Wave but not to be so narrow to the point of affecting coverage. Moreover, larger arrays lead to higher power consumption and time overhead to steer the array beam, which reduces the smartphone's battery life. An $N=4$ linear array's size resulted as a compromise.

The PBS antenna is printed on a $0.5 \mathrm{~mm}$ thick Rogers RT 5880 substrate (with a loss tangent $\tan \delta=0.0004$ and a dielectric constant $\varepsilon_{r}=2.2$ ) and a $2.43 \times 2.43 \mathrm{~mm}$ patch. The metallic ground and patch are made of copper with an electrical conductivity of $5.95 \times 10^{7} \mathrm{Sm}^{-1}$. Since the PBS will be used later for MIMO Spatial Multiplexing performance assessment, it is implemented as a dual polarized antenna, so two feeding pins are placed orthogonally to provide a 
polarization along the $y$-axis and another along the $x$-axis. The PBS was used in a $6.09 \times 24.5 \mathrm{~mm}$ linear array configuration, as seen in Figure 1(a).

The MBS antenna model is a folded $1.4 \times 1.75 \mathrm{~mm}$ monopole, which is considerably smaller than the PBS. It is made out of the same copper material as the PBS. The MBS's ground plane dimensions vary, since this solution will use the mobile phone as a ground plane. It is also designed as a linear array with 4 antenna elements and a $0.7 \lambda$ spacing, displayed in Figure 1(b) that shows the radiation pattern for the $\psi=90^{\circ}$, with an $11.0 \mathrm{dBi}$ maximum gain value. The spacing between antenna elements for both antenna types, $0.7 \lambda$, was determined not only by the desired gain but also by the need to provide good isolation between adjacent elements $\left(s_{i j}<-10 \mathrm{~dB}\right)$.

It is worth mentioning that the patch and monopole array elements used in this study produce the main characteristics that are relevant for quantification of system performance impact analysis, that is, beam agility, array element coupling, and beam depolarization that affect MIMO throughput. Using other antenna elements would not change the conclusions of the study.

Figure 2 shows the angles $\theta$ and $\varphi$, which establish a spherical coordinate system, and also the $\psi$ angle, to indicate the steering direction of the beam [20].

The angle $\theta$ is defined by the $z$-axis and the vector from the origin to the observation point $P$, varying from 0 to $180^{\circ}$. The angle $\varphi$ is defined by $x$ and the projection of $P$ in the $x y$ plane from 0 to $360^{\circ}$. The angle $\psi$ is the elevation beam steering angle. It is measured with respect to the array's axis. Figure 1(a) shows the PBS's $\psi=90^{\circ}$ beam.

\section{Factors Influencing the Antennas' Behavior}

4.1. Form Factor. The smartphone form factor used for CST simulations models an actual phablet as portrayed in Figure 3. This model is mostly a compact structure made out of copper. The form factor has a hollow volume at the bottom, where an FR-4 substrate $\left(\varepsilon_{r}=4.5, \tan \delta=0.025\right)$ was placed, to accommodate the antennas. The bottom area of the phone is encapsulated by a plastic casing, in blue for Figure 3 $\left(\varepsilon_{r}=2.8, \tan \delta=0.0009\right)$. This space is partially occupied by the sub $6-\mathrm{GHz}$ legacy antennas that were terminated by a $50 \Omega$ load in the simulation model, so that their effect would still be accounted for without them being active (since their working frequency bandwidth is different).

The PBS array was integrated into the bottom half of the phone's back, in the volume reserved for the antennas, as seen in Figure 4(a). The MBS was also implemented in the same antenna volume, on top of the phone's PCB, using the phone as a ground plane, as can be seen in Figure 4(b).

It is evident that the radiation patterns suffer some changes by comparing Figure 5 to Figure 1 . This is due to the fact that the phone's chassis that encapsulates the antennas is made of metal. Furthermore, both antenna families have similar maximum gains now, with a small difference of around $1 \mathrm{~dB}$. To study the influence of the mobile phone components on the antenna's radiation pattern behavior, some alterations were made to the original form factor.
Gorilla 5 screen glass [21] $\left(\varepsilon_{r}=6.96, \tan \delta=0.014\right)$ was added to the front of the phone, and the plastic casing was extended all through the back of the phone, to replace the yellow metal rear case. The phone's bezel size was also changed. The bezel is the phone's front frame surrounding the screen. If it decreases to accommodate a bigger screen, it takes space away from the antenna placement volume by covering it with metal. Thus, the bezel size was reduced from its original size of $14.6 \mathrm{~mm}$, displayed in Figure 3, to $2.23 \mathrm{~mm}$, in order to recreate the iPhone $X$ or the Samsung S9 bezel sizes.

The first two modifications to the form factor did not cause any significant changes to the antennas' radiation patterns for this particular antenna placement. This is probably due to the fact that the array was not close enough to the glass for its effects to be manifested. However, the bezel size study confirmed that the addition of metal is detrimental to the propagation of the electromagnetic fields at $\mathrm{mm}$-Wave frequencies, as can be seen from Figure 6. Since changing the form factor size required more metal solely in the front of the phone, the PBS was not severely affected, as represented in Figure 6(a), since it radiates towards the back of the phone. For the MBS, however, the lobe that was directed towards the front of the phone was suppressed as seen in Figure 6(b), but because of the metal's reflective surface, it caused a gain increase in the remaining beam.

However, it is important to stress that all of these results are subject to the arrays' positioning in the phone and should not be generalized. This section is meant to simply identify the most important smartphone impairments to be considered when studying its influence on the antenna's behavior which was, for this scenario, the smartphone's metal chassis. The influence of the other form factor materials was minimal here. Since they would only increase the computation time without affecting too much the results; the rest of the study was carried out with the original form factor in Figure 3.

4.2. User's Hand Grip. A human hand was chosen to model the user's body effect in the performance of mm-Wave antennas implemented in a mobile phone. Intel provided a $3 \mathrm{D} C A D$ of a hand grip, from older projects performed in the sub-6 GHz spectrum. These models can be integrated into CST simulations, and the grip posture can be adjusted to the phablet's dimensions. The hand grip used is commonly known as Talk Mode, representing the way the phone is held when making a phone call, as seen in Figure 7.

Because this CAD model was designed for sub- $6 \mathrm{GHz}$ frequencies, its dielectric properties needed to be updated to $\mathrm{mm}$-Wave frequencies [22]. Although hands are an intricate part of the human body, with skin, bone, muscle, and blood vessels, it was verified that only the muscle tissue is relevant, with the biggest influence in the results. It has the highest values of electrical conductivity, $42.5 \mathrm{Sm}^{-1}$, and dielectric constant, $\varepsilon_{r}=18.63$, out of all the hand components considered in [22] for $39 \mathrm{GHz}$, thus dominating the wave propagation behavior at $\mathrm{mm}$-Wave frequencies. Figures 8 and 9 display the radiation patterns of the arrays implemented in a form factor with the hand grip included for two steering directions, $\psi=90^{\circ}$ and $\psi=70^{\circ}$. 


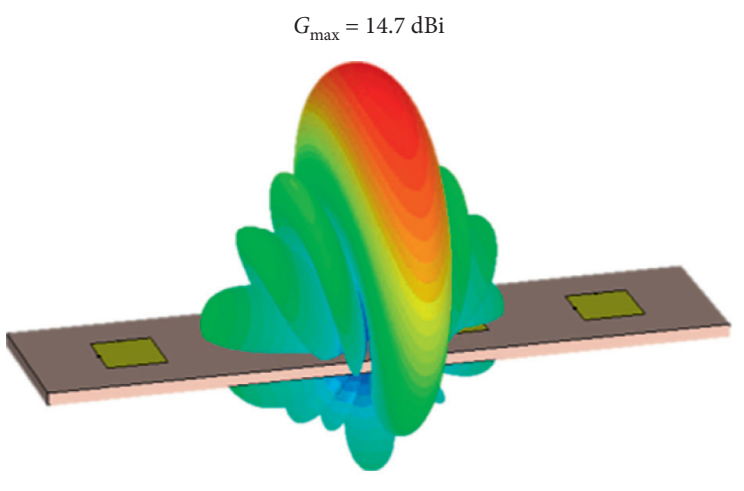

(a)

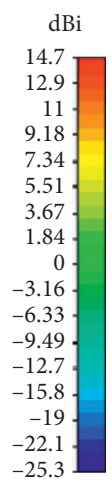

FIgURE 1: Antenna array radiation patterns for $\psi=90^{\circ}$ : (a) PBS; (b) MBS.

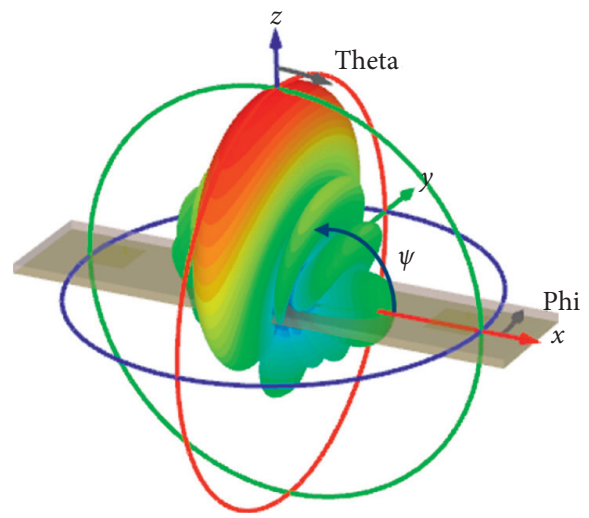

FIGURE 2: Radiation pattern for a linear, four-element PBS array for beam $\psi=90^{\circ}$ to indicate the angles $\theta, \varphi$, and $\psi$.
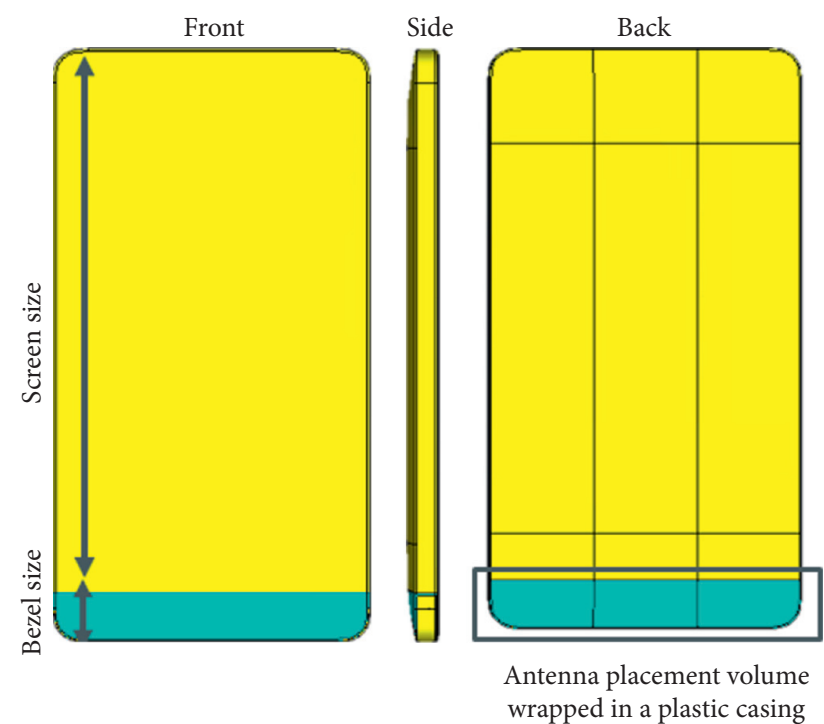

FIGURE 3: Mobile phone form factor with front, side, and back view.

It is clear that all radiation patterns suffer the same effects when in close proximity with the hand. The once narrow and directive beams now take a random and unpredictable shape for their radiation pattern. Considering the transition from the $\psi=90^{\circ}$ beam to the $\psi=70^{\circ}$ beam, it is noticeable that the beam steering ability is also affected since all the beams lose shape when the user is involuntary covering the antennas, losing their directivity. This happens because of the human body's dielectric properties for the mm-Wave band. For such high frequencies, the electric field does not penetrate the human body too deeply (only at the epidermis level) and propagates along the skin's surface.

Figure 10 shows a representation of the E-fields propagation for the PBS over the phone and the user's hand for the hand grip. A transversal cut to the hand + form factor confirms the aforementioned claim. In the place of the user's hand, a big dark blue area is apparent, entailing the lack of electric field penetration. It is either reflected in the user's hand or it escapes from gaps between the user's fingers.

For this particular antenna positioning, the PBS is severely affected since it radiates through the back of the phone that is completely cupped by the user's grip. This is an unavoidable but recurrent challenge that $\mathrm{mm}$-Wave propagation faces and must be diverted through resourceful techniques such as the process of beam switching or array switching. If several antenna arrays are placed in the phone, when the user blocks a certain antenna array with his hand, communications can still be carried out by switching to one of the remaining arrays. However, the topic of array switching alone is very wide and therefore was not explored in this publication.

Additionally, at mm-Wave, the users head also becomes a high attenuation obstacle, and its effect is commonly studied along with the hand as in $[15,23]$. However, since the hand has an equally severe blockage effect, this study focuses solely on the hand grip in order to reduce computation time.

\section{5. mmWAESI's First Step: Coverage Study Step}

Having high gain is not the sole argument to consider in $\mathrm{mm}$-Wave antenna selection for $5 \mathrm{G}$ smartphone integration. There must be a balance between the array's spatial coverage abilities and the value of maximum gain provided. Highly directive beams require beam agility to maintain the link alignment and, consequently, its quality. The underlying 


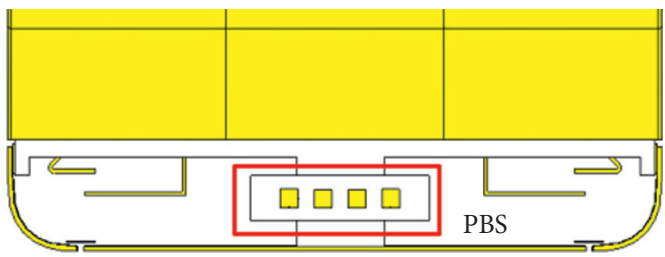

(a)

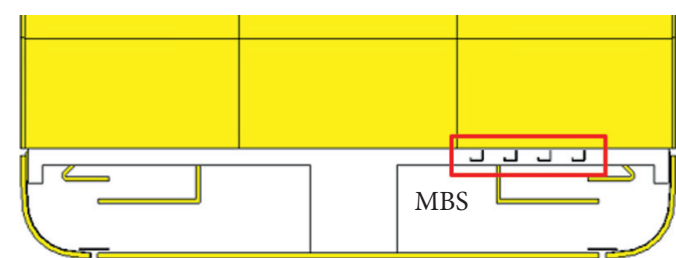

(b)

FIgURE 4: Array implementation in the mobile phone's form factor (plastic casing hidden for better visualization): (a) PBS; (b) MBS.

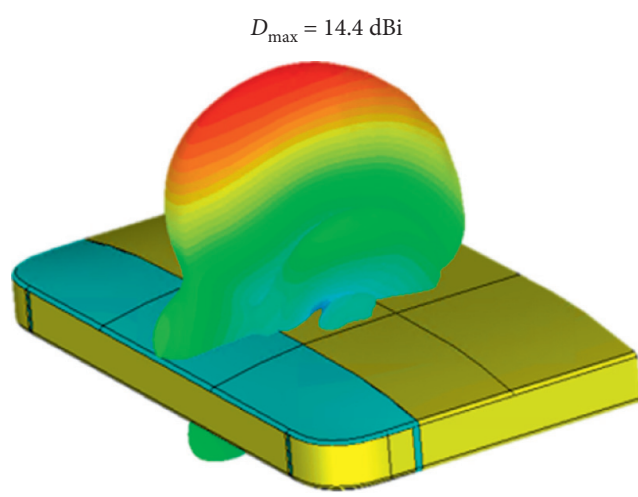

(a)
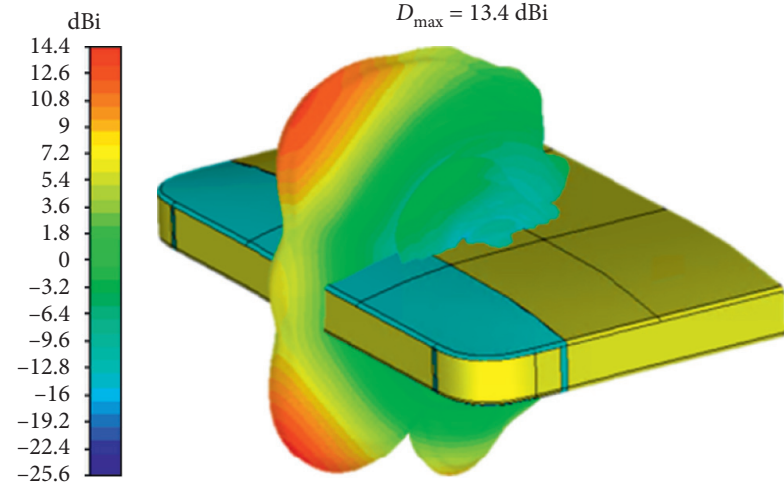

(b)

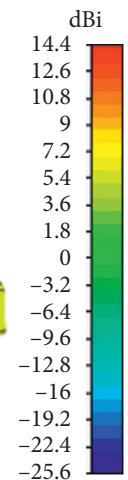

(a) PBS; (b) MBS. principle of arrays is that a single antenna array can provide multiple beams pointing in various directions. To achieve a compromise between gain and coverage, a fair assessment would be to quantify the solid angle that a set of beams can sweep without forfeiting an acceptable maximum gain value. A metric is defined based on the percentage of the total solid angle that the antenna beam can sweep for a given threshold gain, below which the wireless link is not viable.

In order to carry out this study, a tool was developed in MATLAB, the APA TBX, which allows the user to perform the aforementioned evaluation using each of the array's individual antennas' radiation pattern characteristics exported from CST as inputs. The following subsections will detail the underlying principles of this toolbox: the codebook development and the coverage function.

5.1. Codebook Development. The first step in this study is recreating, through simulation, the beam sweep performed by the antenna arrays. This is achieved by computing the array's gain values for each discrete beam in all $(\theta, \varphi)$ directions of observation. Given the analog phase shifters' limitations, it is not possible to perform continuous beam steering. It is a discrete process for specific $\psi$ values.

Therefore, in order to obtain the several $\psi$ beams, a codebook must be developed. A codebook is a set of $M \times N$ weights that are applied to the $N$ individual antennas of an array to generate $M$ beams for different $\psi$ values [24]. For a specific $\psi_{m}$, out of the $M$ available, these $N-1$ weights are defined as $a(m, n)$ and are computed according to linear array theory. Each of the antenna elements will require a progressive discrete phase shift of $\delta$ in its feeding, given by (1), where $k$ is the wave number and $d$ is the spacing between antenna array elements [20].

$$
\delta_{\psi_{m}}=-k d \cos \psi_{m} .
$$

CST has this functionality implemented in the postprocessing tab, under the name of combining tool. The $\mathrm{N}-$ 1 values of $\delta$ must be typed in the feeding of the antennas of the simulation for one beam $\psi_{m}$ to be generated. For a bigger dimension codebook, this can become a tedious and time consuming process, even with the help of a programmed macro.

In order to expedite the process, part of this work's efforts focused on developing the APA TBX tool which makes the codebook generation and representation an automatic process. This tool is configurable, allowing the user to define parameters such as the dimension of the codebook and the phase shifter's resolution and display gain coverage information. CST has an option for exporting the individual antennas' radiation pattern characteristics in the far-field region into an ASCII file (containing not only the mutual influence between antenna elements but also the form factor and the user's influence). These output files are extracted with an angular resolution of $1^{\circ}$ (user selectable). These ASCII files contain, for each $(\theta, \varphi)$ combination, the phase and magnitude components of the electrical field of each antenna element. This information is used in the APA TBX in order to, through linear array theory, generate the array's 

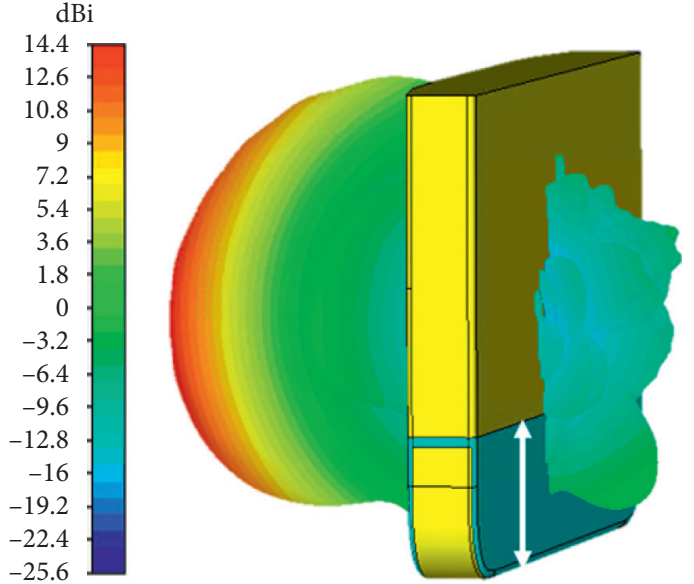

Original bezel size

$\mathrm{dBi}$
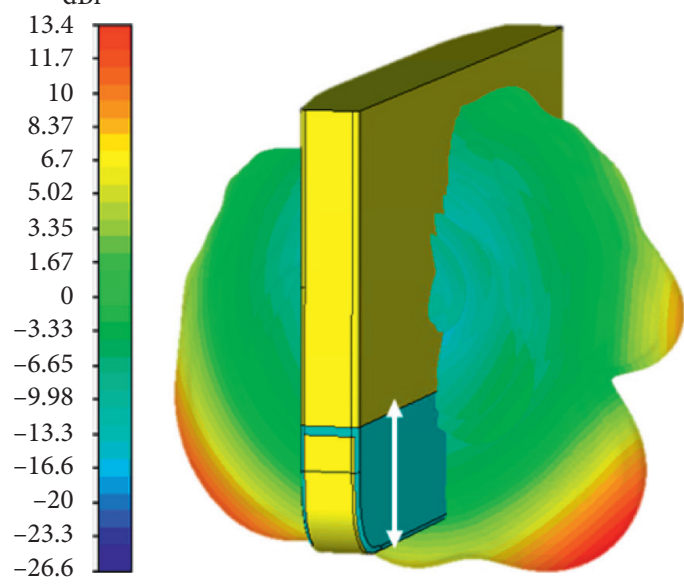

Original bezel size

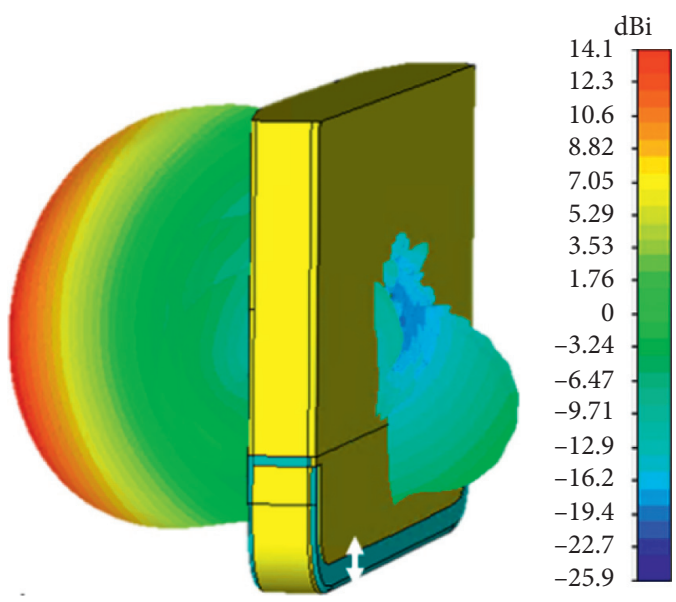

New bezel size

(a)

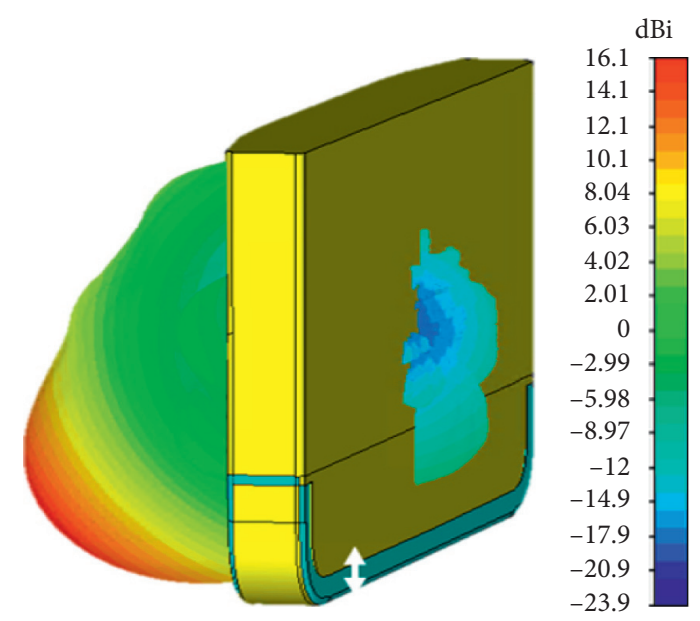

New bezel size

(b)

FIGURE 6: Antenna array radiation patterns for $\psi=90^{\circ}$ with altered bezel sizes. Each plot's colorbar refers to its maximum value with a common dynamic range of $40 \mathrm{~dB}$ : (a) PBS; (b) MBS.

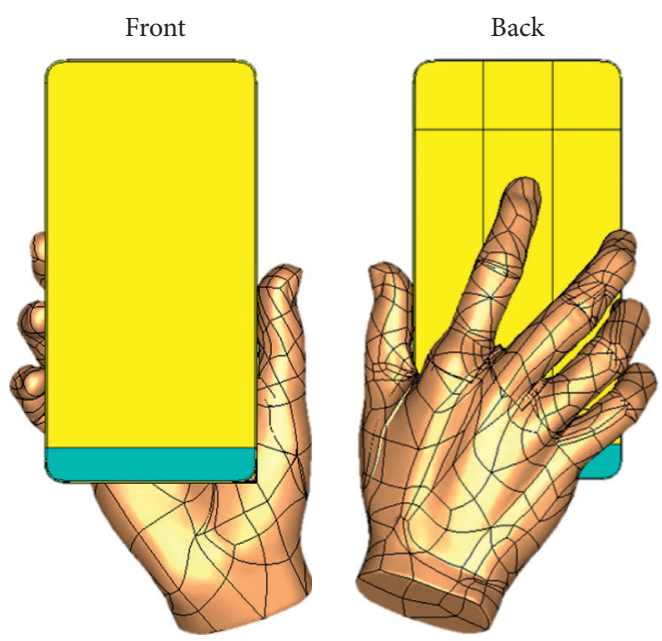

FIgURE 7: Front and back view of the hand grip. 


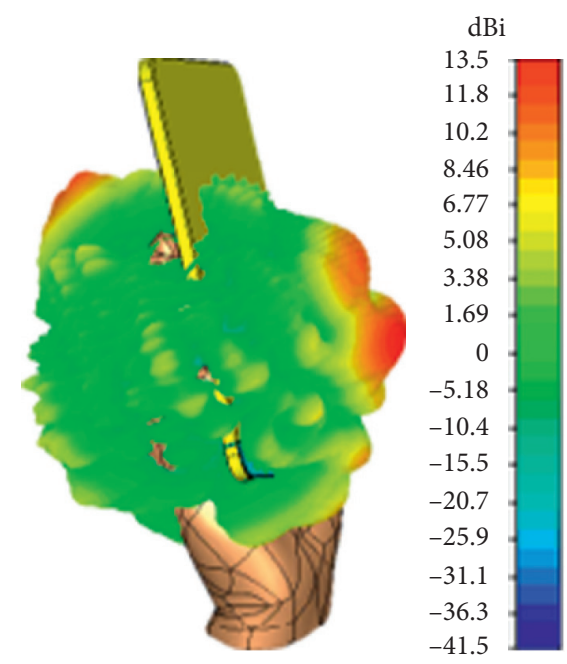

(a)

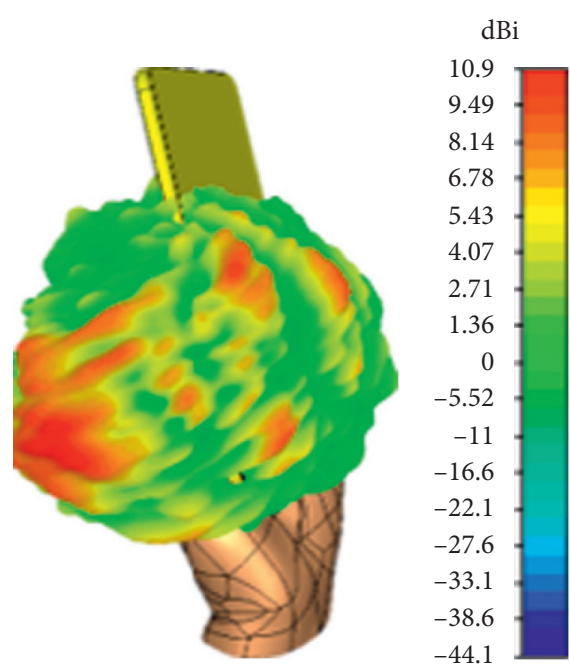

(b)

FIGURE 8: Antenna array radiation patterns for PBS integrated into the form factor with the hand grip. Each plot's colorbar refers to its maximum value with a common dynamic range of $40 \mathrm{~dB}$ : (a) $\psi=90^{\circ}$; (b) $\psi=70^{\circ}$.

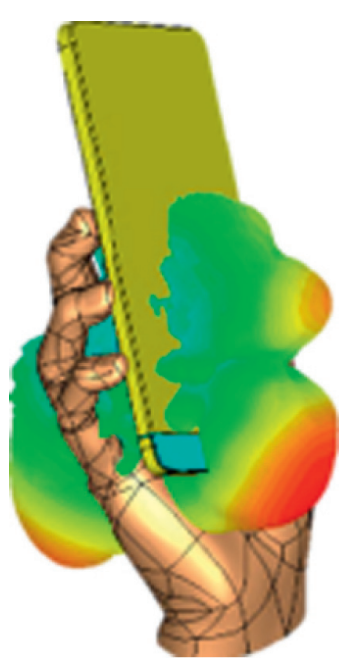

(a)
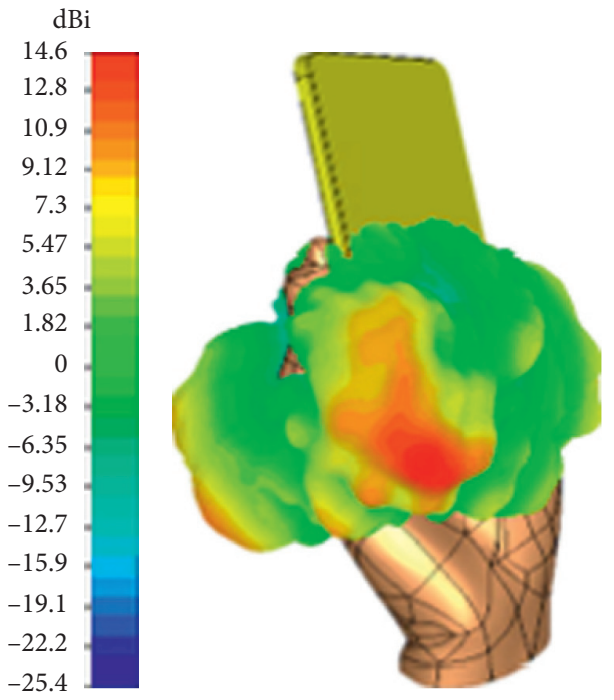

(b)

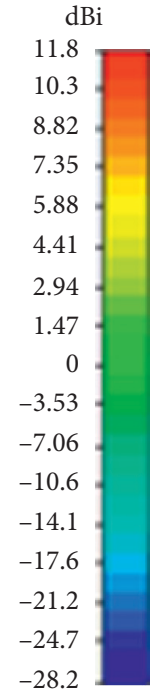

FIGURE 9: Antenna array radiation patterns for MBS integrated into the form factor with the hand grip. Each plot's colorbar refers to its maximum value with a common dynamic range of $40 \mathrm{~dB}$ : (a) $\psi=90^{\circ}$; (b) $\psi=70^{\circ}$.

radiation pattern and compute the array gain according to the user's preferences on array element spacing, number of beams, steering directions, and phase shifter resolution. To use these values for the MIMO portion of this study, a standard linear interpolation for a $2 \mathrm{D}$ function was computed using a MATLAB library to obtain gain values between the discreet $(\theta, \varphi)$ coordinates extracted from CST.

Therefore, the information from these individual files is inputted in the APA TBX, that, with a single simulation, generates and represents a set of beams similar to the ones that would be obtained with the combining tool from CST. Instead of calculating $\delta$ externally and typing it into CST, all that is needed is a list of desired $\psi_{m}$ values, the working frequency, and the antenna element spacing in the array.

Using the APA TBX is quicker and offers more freedom, since functionalities such as bit resolution and phase quantization are included. In this tool, a realistic phase shifter was implemented with a bit resolution input, $B$. For a phase shifter with $B$ bits, there are $2^{B}$ possible phase shifts that can be provided to the antennas. After the $\delta$ values are calculated for each $\psi_{m}$, the tool approximates them to the closest available phase shift. However, if $B$ is too small, the discrepancy between $\delta$ and the closest phase shift available might be high enough to cause a small deviation from the 

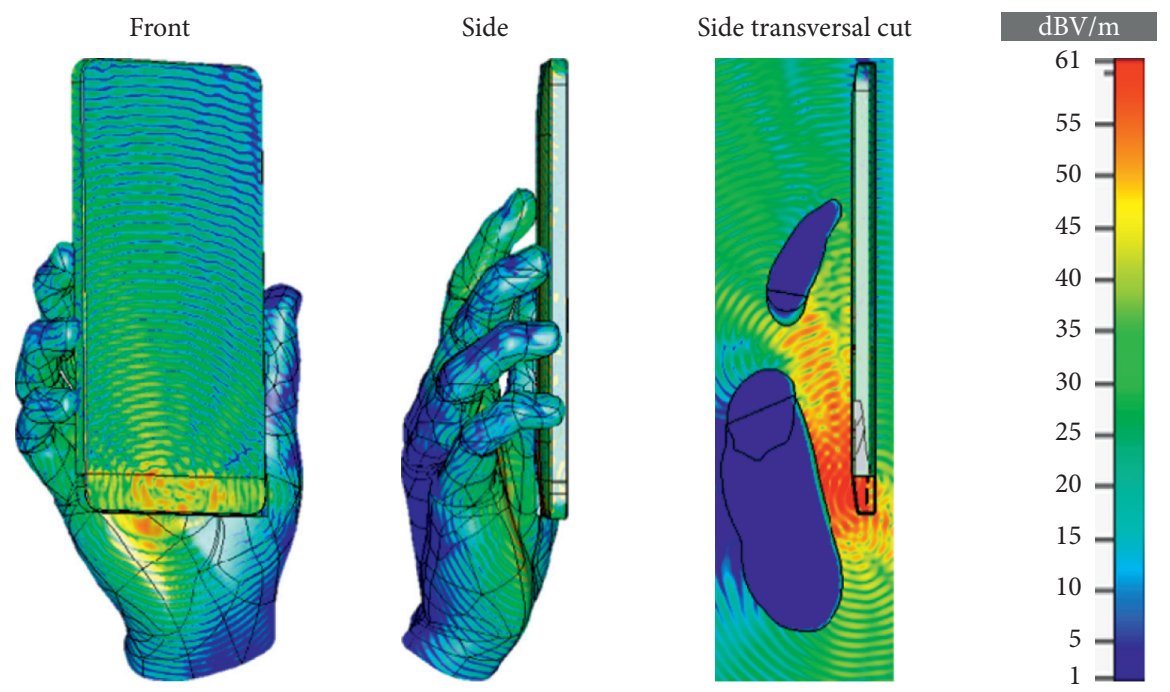

FIgURE 10: E-fields propagation contour plots for the PBS array integrated into the form factor with the hand grip.

originally intended $\psi_{m}$ direction, which is actually a real life limitation of user terminals that requires further computation steps to recreate in CST.

Figure 11(a) shows the four CST individual radiation patterns for a linear MBS array with 4 elements (highlighted in the blue squares) integrated into the mobile phone (suppressed in the figure for better visualization). For the sake of APA TBX validation, the $\psi_{m}=90^{\circ}$ beam is generated in CST's combining tool, in Figure 11(b), and compared to the one obtained from the APA TBX, in Figure 11(c).

This tool also allows for codebook envelope visualization. This is the term used to refer to the representation of the contour of all discrete beams represented simultaneously. Figures 12(a) and 12(b) show the 3D representation of a seventeen-entry codebook envelope for, respectively, the PBS array and MBS array. This codebook envelope will be used to build the coverage function metric that the Coverage Study Step is based on.

5.2. Coverage Function. Consider a spherical representation of an antenna gain in the solid angle, as represented in Figure 13. The center corresponds to the minimum gain value of the radiation pattern, $G_{\min }$, and the radius is the antenna's maximum gain, $G_{\max }$. The 3D codebook envelope of the PBS array is delimited by the coverage sphere. Suppose that a certain gain threshold value is established, $G_{\text {threshold }}$, under which the gain is too low for the antenna to be used. The coverage function expresses the percentage of registered directions $(\theta, \varphi)$ of the codebook envelope that are within that gain interval, $G_{\text {threshold }}<G<G_{\max }$. This percentage will quantify the full gain coverage potentiality of each antenna solution.

However, when exporting data for a radiation pattern, CST samples the data for equally spaced points of the radiation pattern in $\widehat{\theta}$ and $\widehat{\phi}$ directions. This creates a $180 \times 360$ grid, with uniformly distributed points for each direction. In spherical coordinates, this does not correspond to a uniform distribution of points over the sphere. The nodes become denser as $\theta$ approaches 0 or $180^{\circ}$ and sparse when approaching $90^{\circ}$ as can be seen in Figure 14. This would mean that the percentage of points abiding the $G_{\text {threshold }}<G<G_{\max }$ condition would be dependent on the orientation of the radiation pattern.

A weight function must be added to properly account for the power density distribution in a polar grid from rectangular grid data. The weight function, $\mathrm{pc}_{w}$, is given by (2). Areas with higher point density will weigh less than areas where the points are sparser.

$$
\mathrm{pc}_{w}(\theta)=\frac{\sin \theta d \theta d \phi}{4 \pi} .
$$

This will then be used to create a curve representing the coverage percentage, Coverage $e_{\%}$, for $L$ levels of $G_{\text {threshold }}$. This calculation is described in (3), where $N_{G_{\text {threshold }}}$ is the number of points that are above $G_{\text {threshold, }}, \mathrm{pc}_{w}$ is the corresponding weight value according to the points' $\theta$ coordinate, and $P$ is the total number of points analyzed.

$$
\text { Coverage }_{\%}=\frac{\sum_{n=1}^{N_{G_{\text {threshold }}}} \mathrm{pc}_{w}\left(\theta_{n}\right)}{P} .
$$

\section{6. mmWAESI's Second Step: MIMO Performance Step}

A 3D geometric discrete scattering channel model was implemented to recreate different types of scenario for $\mathrm{mm}$ Wave propagation.

The channel model was idealized for a MIM$\mathrm{O}+$ beamforming scenario with Spatial Multiplexing. For this particular study, a $2 \times 2 \mathrm{MIMO}+$ beamforming system is considered, such as the one in Figure 15. Therefore, the UE has now two arrays in the bottom back of the phone, $A_{\mathrm{UE} 1}$ and $A_{\mathrm{UE} 2}$, and the $\mathrm{BS}$ has also two arrays, $A_{\mathrm{BS} 1}$ and $A_{\mathrm{BS} 2}$. The two pairs of arrays create four individual transmission paths, each one of them with complex transmission coefficients, $h_{11}, h_{12}, h_{21}$, and $h_{22}$. Once put together, these coefficients form a $2 \times 2$ channel matrix $H$ that summarizes in matrix 

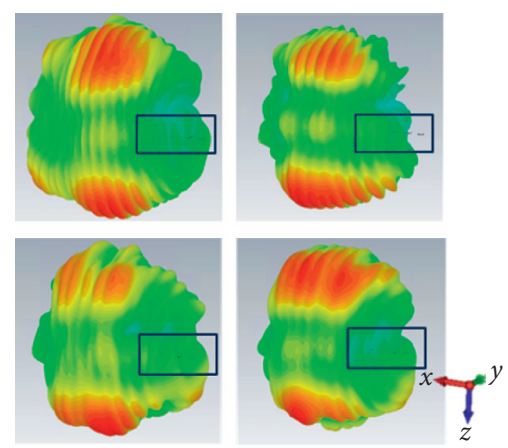

(a)

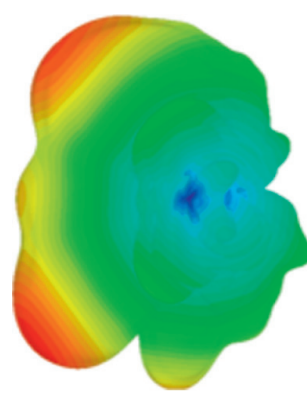

(b)

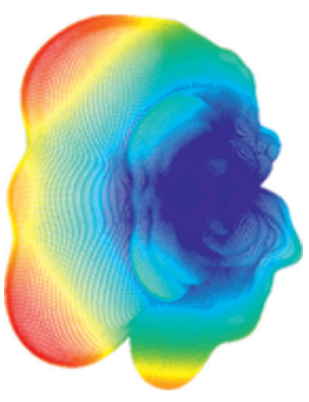

(c)

FIgURE 11: Codebook development process for $\psi=90^{\circ}$ : (a) individual CST MBS radiation patterns; (b) CST; (c) APA TBX.

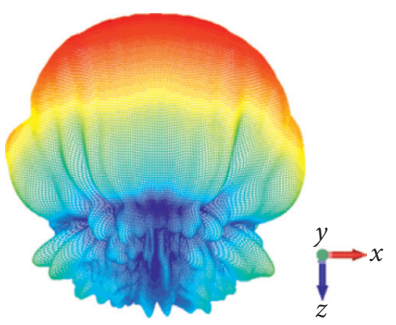

(a)

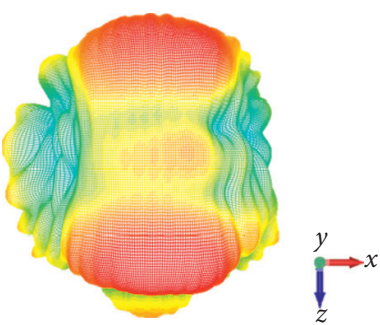

(b)

FIGURE 12: 3D 17-entry codebook envelope representation: (a) PBS; (b) MBS.
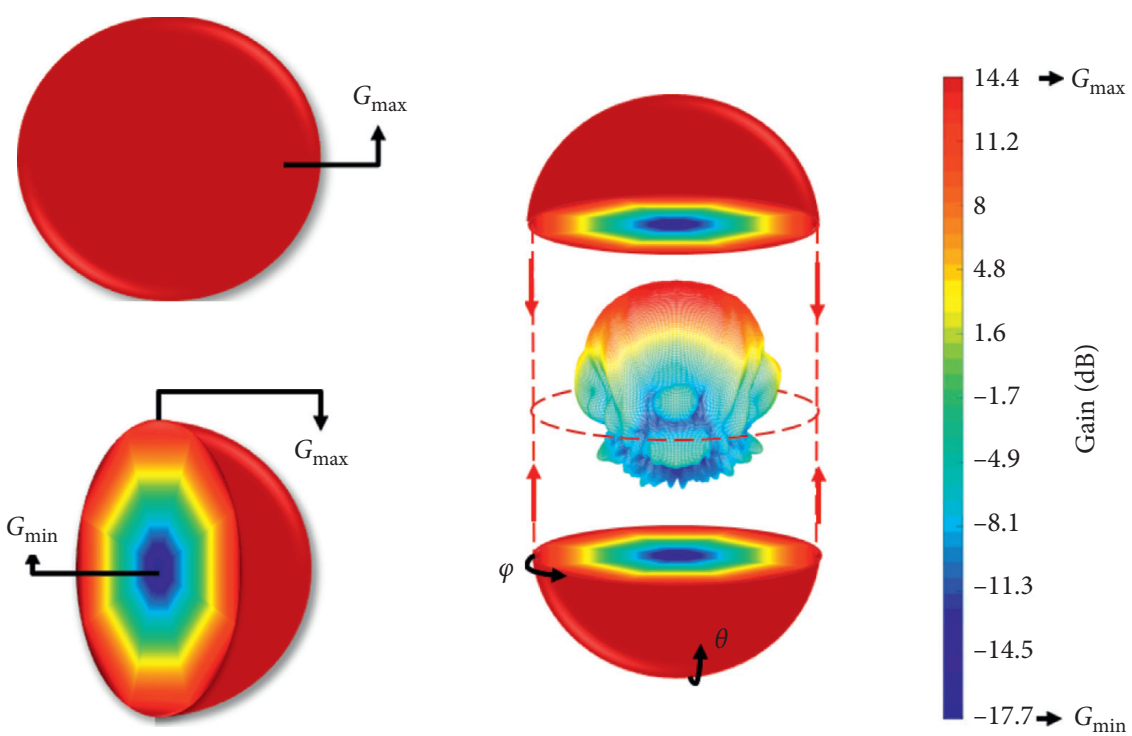

FIGURE 13: Coverage function methodology applied to the 17-entry codebook envelope of the PBS array.

form the current channel state in response to the environment [25].

The BS's arrays, $A_{\mathrm{BS} 1}$ and $A_{\mathrm{BS} 2}$, spaced by a $2.5 \mathrm{~cm}$ gap, are two linear, four-element PBS arrays with orthogonal polarizations, one over the $x$-axis and another over the $y$-axis. $A_{\mathrm{UE} 1}$ and $A_{\mathrm{UE} 2}$ are integrated into the back of the phone and can be either PBS or MBS arrays.
For the PBS, the two arrays consist of one dual polarized PBS array, as shown in Figure 4. For the MIMO Performance Step, in order to evaluate the Spatial Multiplexing performance, a second array is added to the mobile phone's form factor. Due to lack of space for an orthogonal polarization arrangement, the arrays are placed symmetrically in the mobile phone, as seen in Figure 16, horizontally and with the 


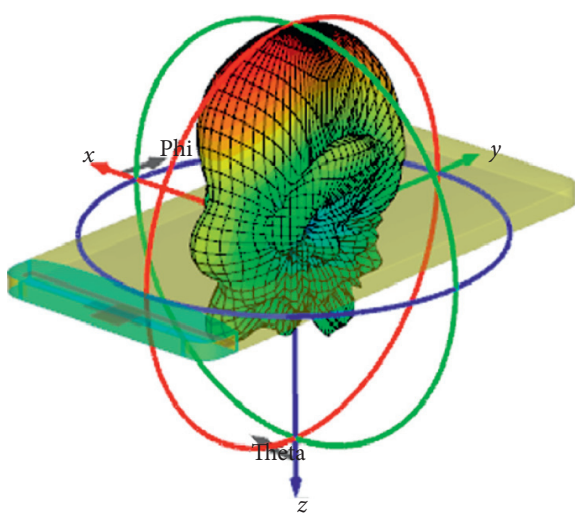

(a)

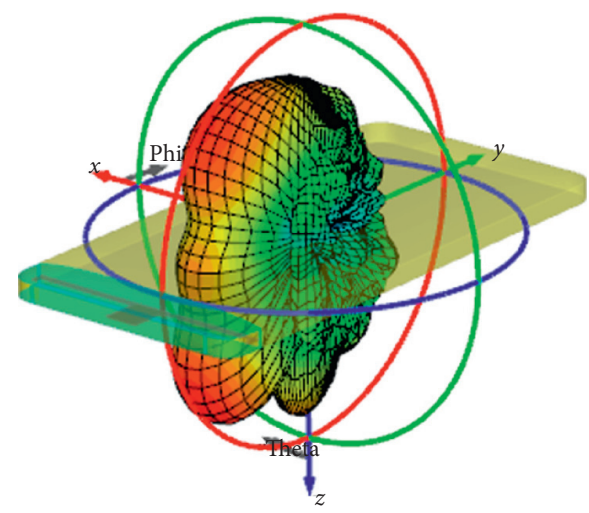

(b)

FIgURE 14: CST's point density: (a) PBS in the form factor; (b) MBS in the form factor.

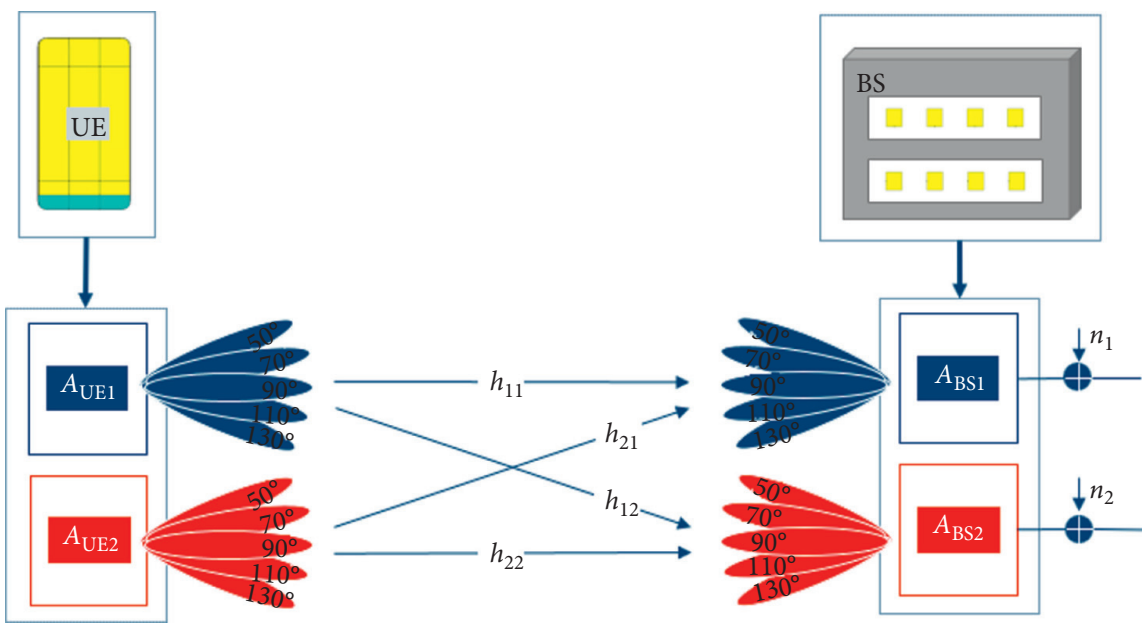

Figure 15: Schematic view of idealized MIMO + beamforming system for the simulator.

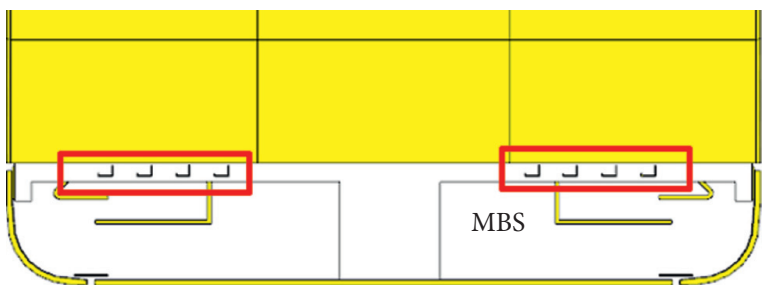

FIgURe 16: Chosen MBS antenna placement for MIMO performance evaluation.

same orientation. Because of this, it is anticipated that, for a predominantly LOS environment without any multiple paths, the MIMO performance might deteriorate to SingleIn-Single-Out (SISO) performance.

The number of beams generated has been set to five codebook entries, $M=5$. These are $\psi=50^{\circ}, \psi=70^{\circ}$, $\psi=90^{\circ}, \psi=110^{\circ}, \psi=130^{\circ}$. For this study, the UE and BS are separated by a distance $d_{\mathrm{LOS}_{\mathrm{UEBS}}}=120 \mathrm{~m}$ and surrounded by $S=15$ scatterers distributed randomly over an area of
$12 \times 140 \mathrm{~m}^{2}$. The number of scatterers was carefully used so that there would be enough multiple paths to recreate Rayleigh fading. These scatterers mimic every day obstacles in an urban area, as seen in Figure 17, and move randomly along the $x$-axis over time with a $2 \mathrm{~m}$ range of motion. Moreover, they induce a Gaussian distribution of polarization rotation and reflection factor transition, being responsible for creating enough reflections of the signal to successfully create multiple paths in the MIMO channel.

This channel model uses realistic antenna characteristics as well as the studies made in the previous section regarding form factor influence, beamforming, user inherent body blockage, and user induced phone rotation. It will be used to determine the channel's performance for each antenna type when the UE is subjected to different environment scenarios, orientations in space, and hand grips.

6.1. H Matrix Calculation. In a typical urban environment, there are two signal propagation types. On the one hand, there is the direct LOS, when the transmitter and the receiver have an unobstructed path between them. On the other 
hand, there is propagation through multiple paths, where, given the presence of scatterers, the signal is reflected and scattered in multiple directions only to arrive at the receiver through several different paths and with different phase shifts. While many studies in the literature use a statistical distribution, such as the Rayleigh distribution, to generate the multipath components, in the present study the multipath components are generated from true scatterers distributed in the scenario, each one with changing position, scattering amplitude, and scattered depolarization over simulation time. The scatterers move along linear paths with controllable speed. Their scattering amplitudes follow a Gaussian distribution with zero mean and settable standard deviation ( 0.55 in the present study). The depolarization angle follows a uniform distribution in a settable interval. It is worth mentioning that, in this study, although the scatterer's amplitude follows zero-mean Gaussian distribution, the reflected path's amplitude accounts for each scatterer's uniformly distributed depolarization factor and its $1 / d_{S_{i}}$ distance dependence in space, where $d_{S_{i}}$ is the total distance from BS to the scatterer $S_{i}$ to the UE. Therefore, the scatterers' reflection amplitude is no longer zero-mean Gaussian distributed and, consequently, not Rayleigh distributed in the general case. Moreover, the $\mathrm{H}$ matrix calculation includes path loss by establishing a dependency of $1 / d_{S_{i}}$ for all scattering paths. Since this model accounts for the antenna radiation pattern, UE rotation angles, beam shape modification by user influence, and angles of arrival and departure, it allows for a more realistic characterization of the scenario, while replicating canonical channels such as Rayleigh, if necessary for benchmarking purposes. Therefore, in order to obtain the channel state matrix, both types of components are recreated in the simulator and considered in the calculations, as seen in

$$
H=H_{\mathrm{LOS}}+H_{\text {multipath }} \text {. }
$$

The $H$ matrix will be created for a user-defined set of environment conditions during a large number $T$ of time slots, in order to create a fading distribution that can be evaluated. Because all beam combinations, $c$, between the BS array and the UE array will be taken into consideration, the $H$ matrix (with dimensions $2 \times 2 \times T$ ) will be calculated $c$ times. A dedicated beam selection algorithm will then be applied to choose the $H$ matrix that corresponds to the most fitting combination. This matrix will then be put through the channel assessment metrics that will evaluate the channel's performance for a specific antenna family.

6.2. Phone Rotation. While using the phone, the user will involuntarily change its orientation relative to the base station. By rotating the phone, the polarization of the UE's signals arriving at the BS will change, affecting the MIMO channel's performance.

Therefore, in order to grasp the full effect of UE rotation in the MIMO channel, it is important to observe the UE rotation in all 3 axes, $x, y$, and $z$. CST's far-field plot properties allow for Local Coordinate System (LCS) selection (also called Working Coordinate System in CST, or
WCS) for radiation pattern observation. Using one radiation pattern calculation, it is possible to mimic any other phone position with respect to the reference axis frame to extract the corresponding E-field data to use in the simulator for the $H$ matrix construction.

A total of 18 phone positions were chosen, separated by a $45^{\circ}$ rotation step in all three axes, as seen in Figure 18(a). These positions are meant to represent the range of motion of the mobile phone, as can be seen from Figure 18(b).

6.3. Array Coupling. Another influencing factor on the MIMO channel performance is the coupling between arrays in the same device [17]. If the coupling between the two arrays is high, this means that the arrays are too close and there is energy waste that could be applied to signal transmission. In addition, if part of an array's energy goes into the other array, some of its information gets misdirected, resulting in crosstalk and loss of MIMO gain. In order to evaluate this correctly in the model, the array's feeding network must be defined, so that each array is represented by one single port using CST's circuit analysis tool, as seen in Figure 19. This will return a $2 \times 2 S$-parameter matrix that will allow us to calculate the channel's $2 \times 2$ transmission matrix $T$ that quantifies the amount of existing crosstalk.

Simulations with the full form factor showed that, for the PBS and MBS array placement in the smartphones adopted in this work, the diagonals of the $T$ matrix came very close to one and the crosstalk entries were close to 0 (in linear units). This means that there was no significant array coupling to account for in the $H$ matrix.

6.4. Beam Pair Link Selection. The beam pair selection criterion is based on two factors: Received Power and Condition Number $(\mathrm{CN})$. For each $h_{i j}$ channel connecting $A_{\mathrm{UE} i}$ to $A_{\mathrm{BS} j}$, a matrix is calculated over time with the power levels for each beam combination, $c_{i j}$, as seen in Figure 20. The entries with the highest power levels are then compared according to their $\mathrm{CN}$. The $\mathrm{CN}$ is a mathematical indicator, obtained from the $H$ matrix, that assesses the MIMO channel capability of supporting Spatial Multiplexing. Since the Spatial Multiplexing transmission technique is based on simultaneous transmission of independent and separate data streams, it is necessary to evaluate how sensitive the receiver arrays are to noise and measurement errors and how much that will compromise data recovery. According to [26], in linear units, an ideal transmission would have $\mathrm{CN}=1$. For $\mathrm{CN}>10$, the channel is also considered unsuited for Spatial Multiplexing. Therefore, the $c_{i j}$ chosen will correspond to the matrix entry whose $\mathrm{CN}$ is closest to 1 .

6.5. MIMO Channel Capacity. The channel capacity is the main metric chosen to evaluate the MIMO channel performance. Equation (5) expresses Shannon's capacity theorem [27]. $N_{t}$ and $N_{r}$ represent, respectively, the number of transmitting and receiving arrays of the system, while $I_{N_{r}}$ is an $N_{r} \times N_{r}$ identity matrix, $H_{n}$ is the normalized channel 


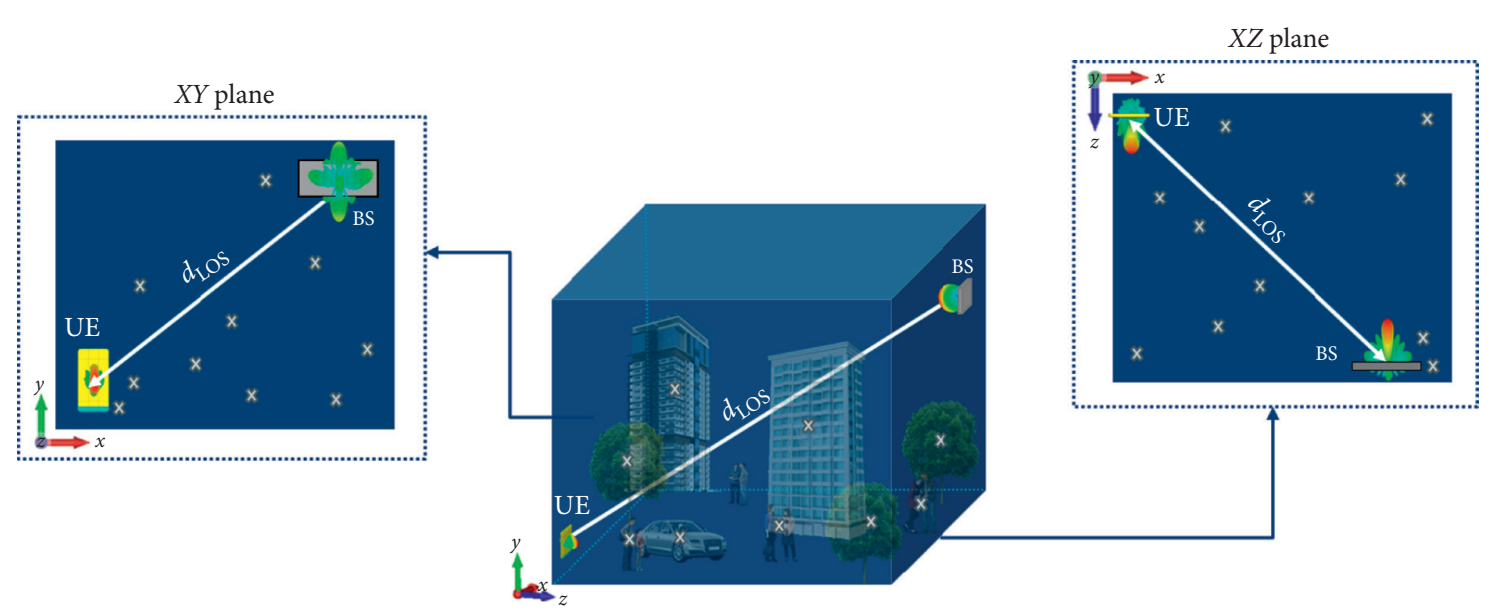

FIGURE 17: Scatterer depiction in the channel model.

matrix of the system, and SNR is the receiver's signal-tonoise ratio. For a $2 \times 2 \mathrm{MIMO}$ scenario, $N_{t}=N_{r}=2$ and $H$ is a $2 \times 2$ matrix.

$$
C=\log _{2}\left(\operatorname{det}\left[I_{N_{r}}+\frac{\mathrm{SNR}}{N_{t}} H_{n} H_{n}^{H}\right]\right) \mathrm{bit} / \mathrm{s} / \mathrm{Hz} .
$$

It is important to mention that this calculation of $C$ gives the best case scenario value of the capacity, since it only takes into consideration the channel conditions and ignores processes such as modulation/demodulation and coding/ decoding of the signal.

Regarding the SNR, many MIMO models fix this metric at a reasonable value, generally 10 to $20 \mathrm{~dB}$. However in this simulator, the SNR is intentionally calculated over time using the link power budget and is susceptible to the change of environment characteristics and distance between the antennas.

\section{Results}

7.1. mmWAESI's First Step: Coverage Study Step. Figure 21(a) displays the PBS and MBS coverage curves versus $G_{\text {threshold }}$ for the smartphone implementation displayed in Figure 4 with Figure 12's codebooks. Similar to a Cumulative Distribution Function (CDF) representation, these curves represent the Coverage $e_{\%}$, or, in other words, the solid angle percentage of the UE antenna, where its gain is above the stipulated $G_{\text {threshold }}$ value.

The phase shifter was configured to have a 5 bit resolution and $360^{\circ}$ range. $G_{\max }$ corresponds to the highest gain value of each of the two codebook envelopes. In this case, $G_{\text {max }_{\mathrm{PBS}}}=14.33 \mathrm{dBi}$ and $G_{\max _{\mathrm{MBS}}}=14.08 \mathrm{dBi}$. The curve starts at $100 \%$ because all the evaluated gain values are higher than $G_{\text {threshold }}=G_{\min } \approx-25 \mathrm{dBi}$. The plot shows that the MBS presents higher gain in a larger percentage of the solid angle compared to the PBS. Despite having similar maximum gain values, the MBS array has a wider codebook envelope shape that extends in all directions. The PBS, on the other hand, radiates in a slightly more concentrated number of directions, which explains why when the threshold value passes the curves' crossing point, the PBS takes the lead. This reiterates the delicate balance between coverage and gain.

These tests were repeated to include the hand grip, in order to account for the user's hand blockage effect. Figure 21(b) shows that, for this antenna placement, adding in the user's hand makes the coverage curves get even closer to each other. This is due to the fact that, as seen in Section 4, the hand alters the radiation patterns, tending to take a random shape with a certain gain value. This suggests that, for this particular single array integration scenario, there is no significant performance difference between using PBS or MBS when all environment components are taken into account. A possible solution, relevant for future publications, would be multiple-array implementation in the smartphone to perform array switching.

7.2. mmWAESI's Second Step: MIMO Performance Step. To test the MIMO channel performance sensitivity to phone rotation, as described in 6,18 different phone orientations were used, with and without the hand grip, to calculate the MIMO channel's capacity distribution over the 3D space. This evaluation took place under three different test scenarios: LOS, LOS + multipath, and NLOS + multipath, as represented in Figure 22.

7.3. MIMO Channel Capacity. This section presents the phone-rotation capacity outage results in the format of CDF curves. As seen in Figure 23, the $y$-axis displays the percentage of UE orientations that achieve values less than the abscissa value. From the three environments tested, the one that presented the highest data rate values was the LOS + multipath, Figure 23(b), followed by NLOS + multipath, Figure 23(c), and finally, LOS Figure 23(a). These results confirm that the presence of scatterers in the environment results in an increased MIMO channel performance. This is because MIMO thrives in multipath rich environments, since several paths are available for the signals to propagate through.

Moreover, by evaluating the median values of these channel capacity distribution over the UE orientation, it is 


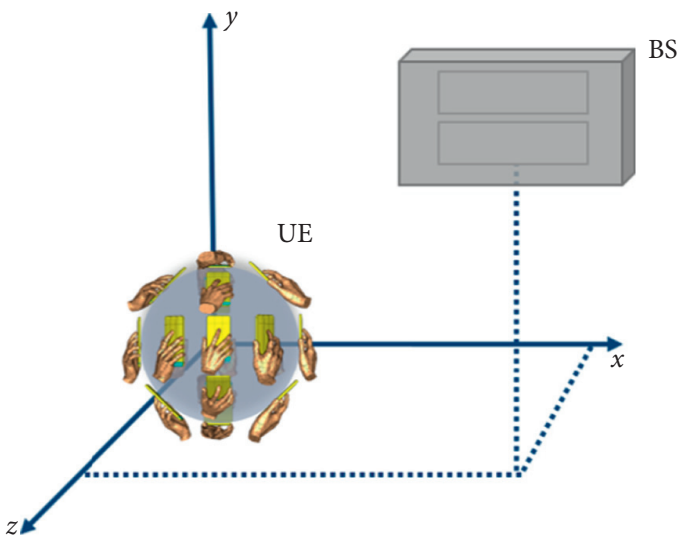

(a)

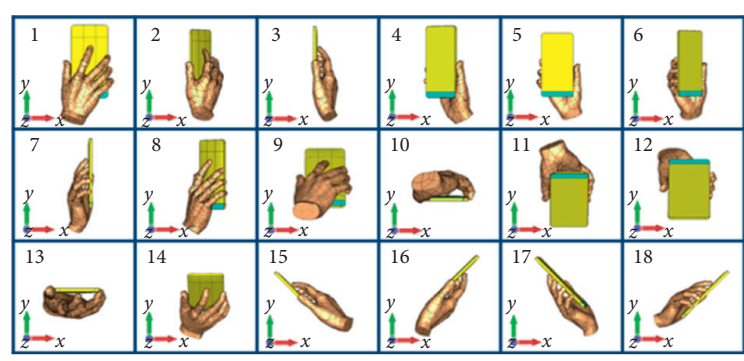

(b)

FIGURE 18: Introducing phone rotation in the system: (a) 3D representation of the rotation modes with relation to the BS; (b) nomenclature for the 18 rotation positions.

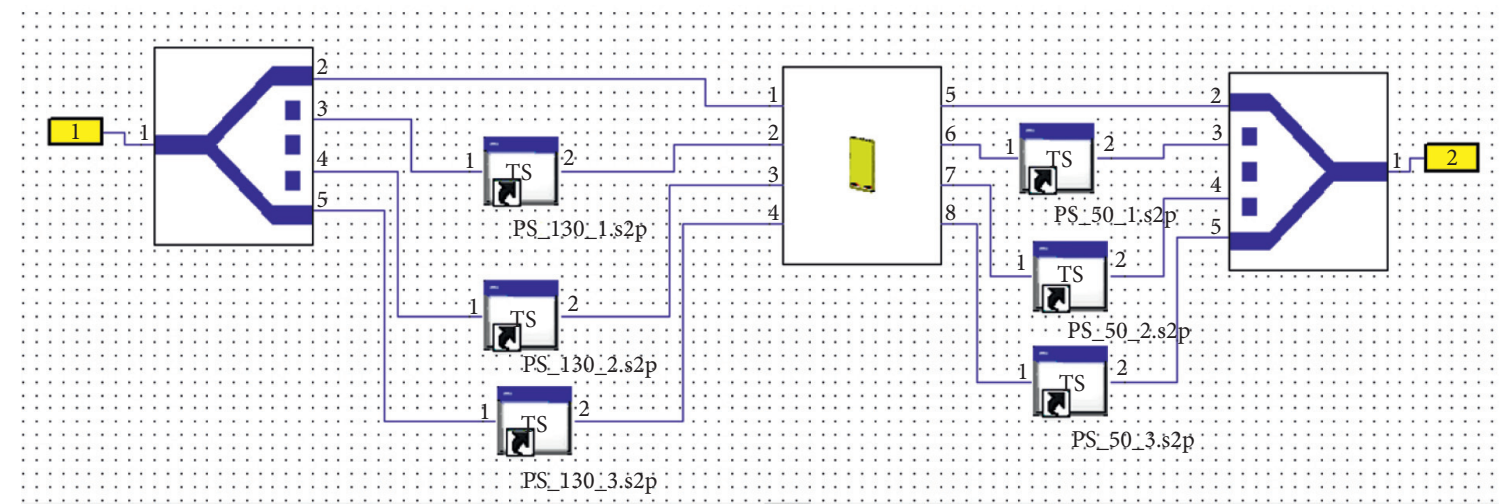

FIGURE 19: CST schematic view of the circuitry used for obtaining the array coupling values between the two UE's MBS arrays. Each yellow box is a single port representing one of the 4-element arrays. The squares are the phase shifts applied to each antenna of the array for a particular $\psi_{m}$.

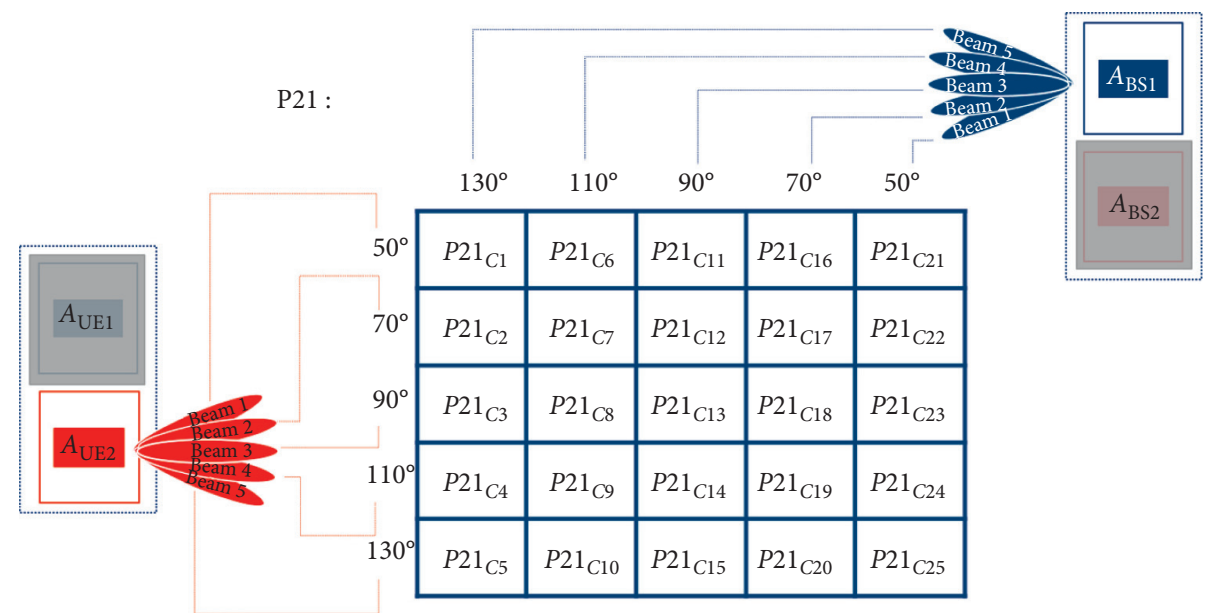

Figure 20: P21, a particular power matrix for the $h_{21}$ channel for time slot $t$.

possible to conclude that, for this single antenna array integration scenario, the MBS and PBS results are similar, especially when considering the hang grip influence. This reveals, once more, the weight of user blockage from a MIMO performance standpoint and also the need to implement multiple antenna arrays in the UE as a counteraction. 


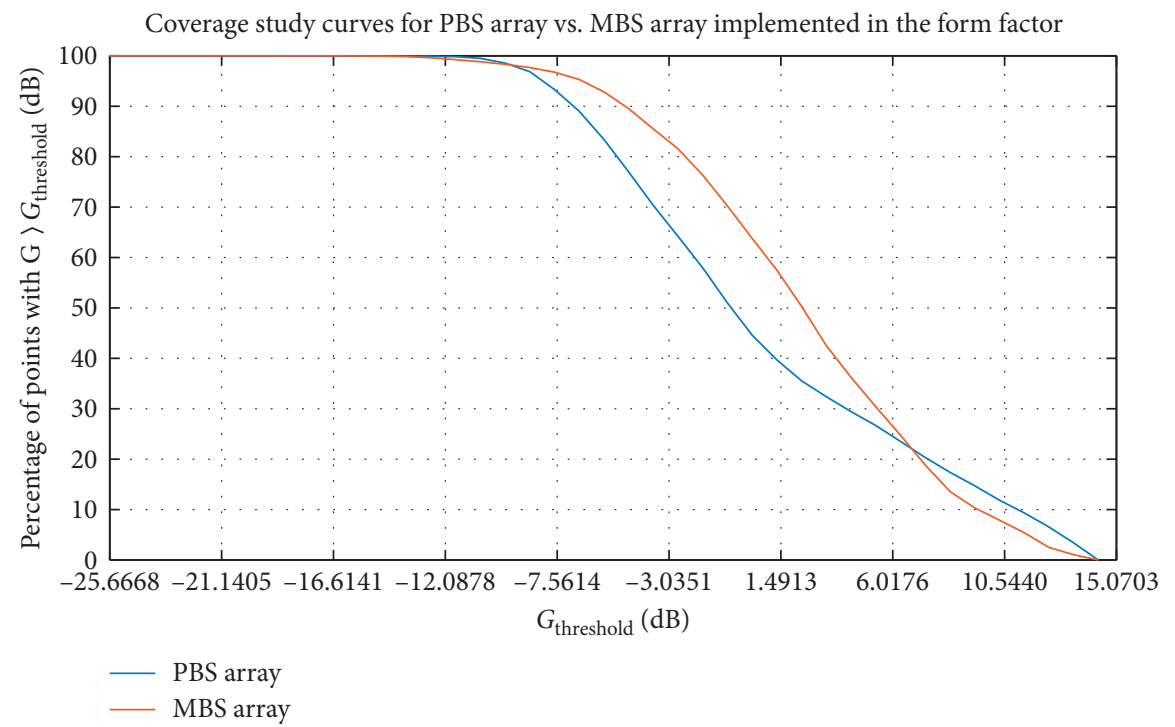

(a)

Coverage study curves for PBS array vs. MBS array implemented in the form factor with hand grip TM

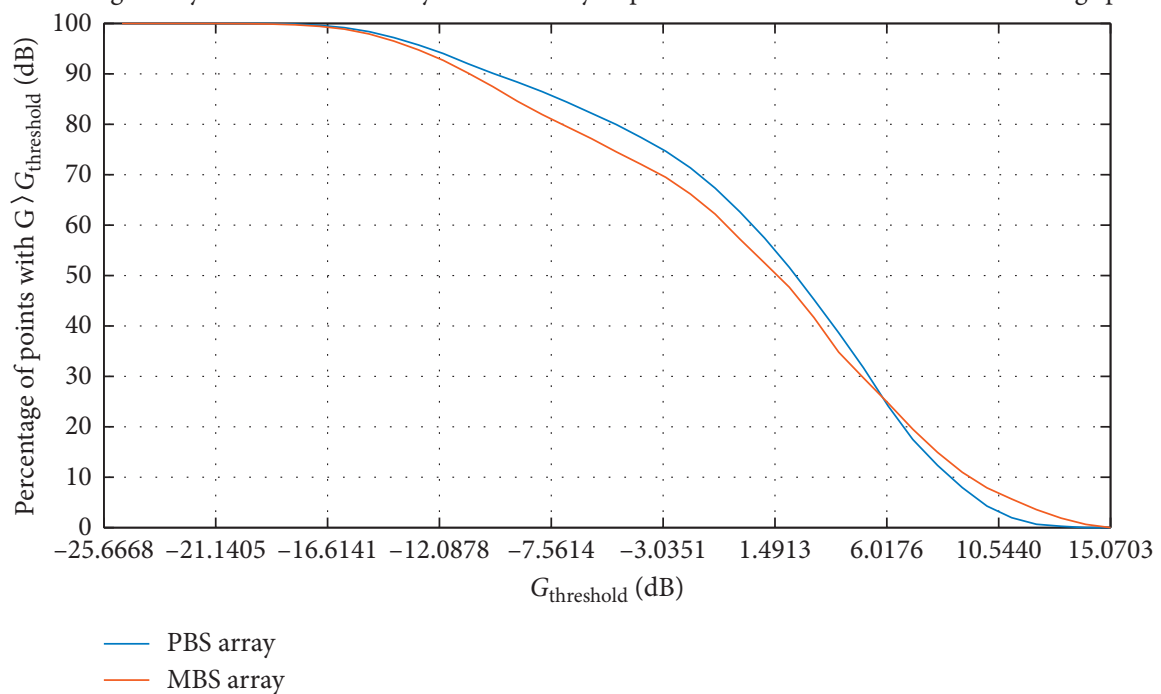

(b)

FIGURE 21: Coverage curves for PBS vs. MBS implemented in the smartphone (TM: Talk Mode hand grip): (a) without user presence; (b) with hand grip.

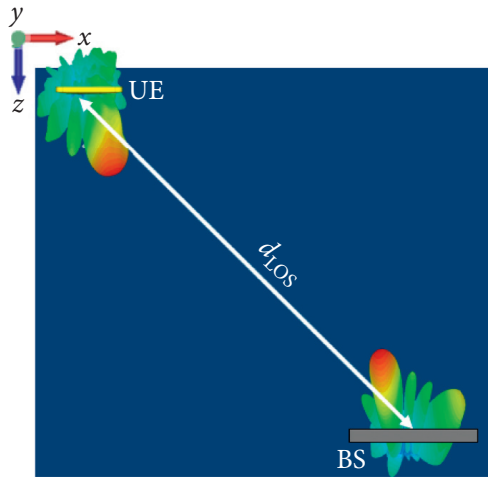

(a)

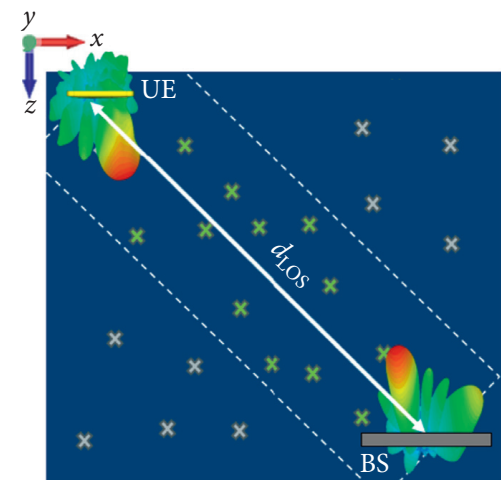

(b)

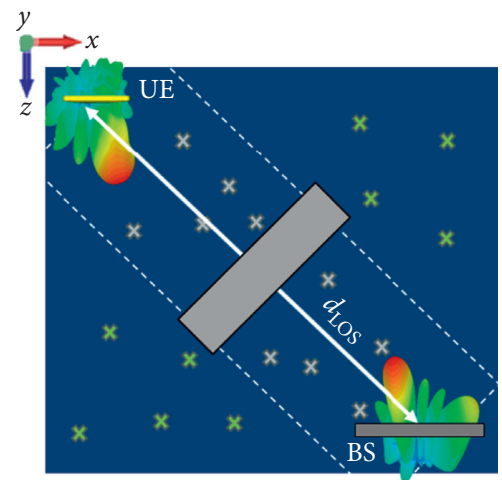

(c)

Figure 22: Representation of the three test scenarios in the $x y$ plane (MP: multipath): (a) LOS; (b) LOS + MP; (c) NLOS + MP. 


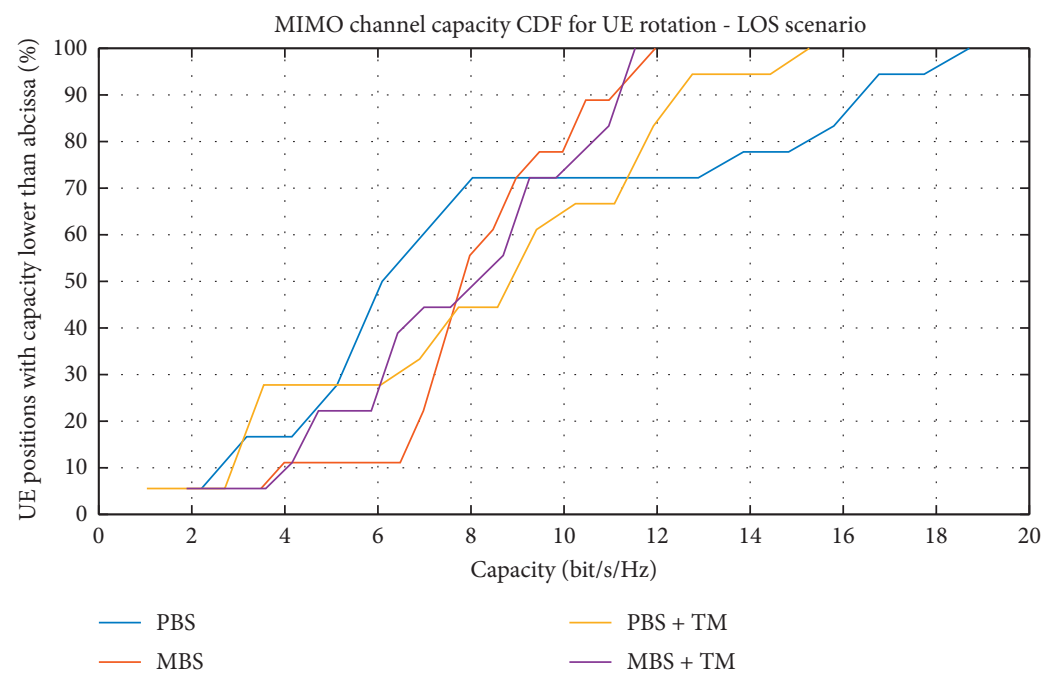

(a)
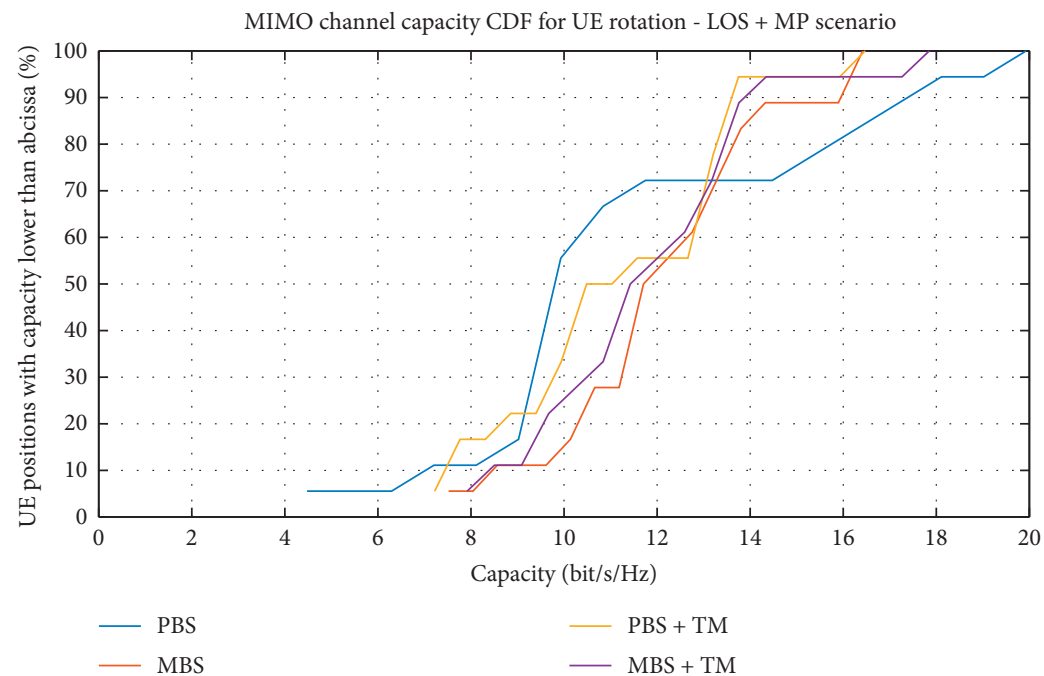

(b)

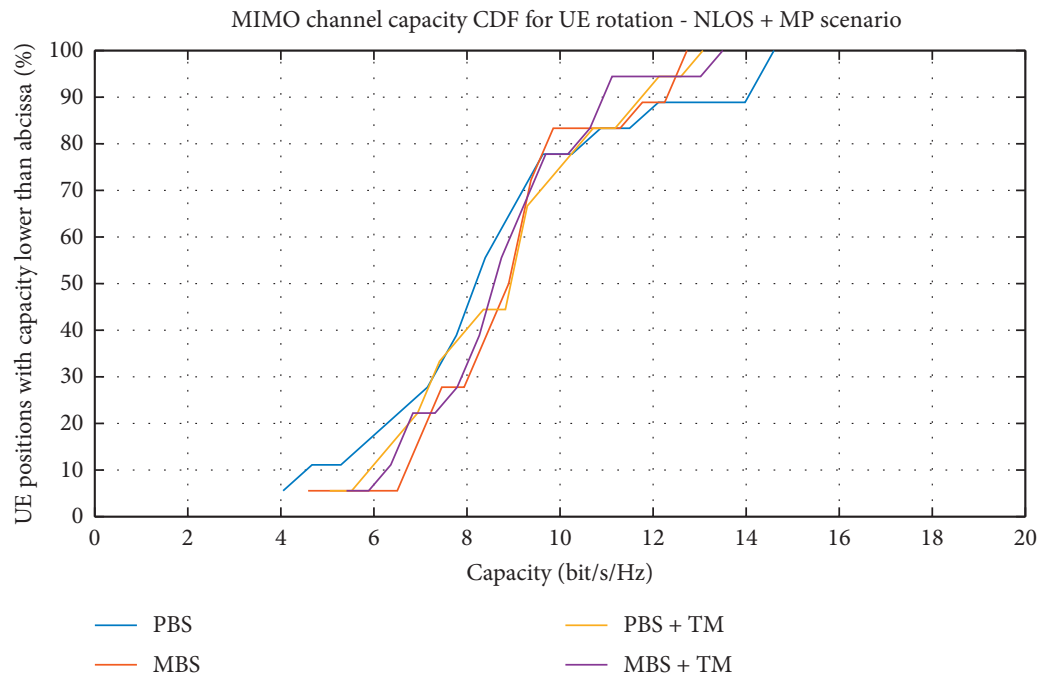

(c)

FIGURE 23: Capacity CDF according to the UE positions for PBS, MBS, PBS + hand grip, and MBS + hand grip (TM: Talk Mode hand grip): (a) LOS; (b) LOS + multipath; (c) NLOS + multipath. 
It is also worth mentioning that although analog beamforming is considered in this study, there are also other approaches regarding beamforming architecture. While analog beamforming is selected given its energy-efficient nature, it is also restrictive, since it can only produce one beam at a time, potentially affecting the capacity performance studied in this section. On the other side of the spectrum, digital beamforming allows multiple beams to be formed simultaneously, which would increase MIMO capacity by providing different simultaneous transmission paths. However this solution also causes massive energy consumption that the UE may not be able to sustain. The compromise may reside somewhere between digital beamforming with low-resolution and hybrid beamforming, which is an alternative beamforming solution that provides a higher number of simultaneous beams than analog beamforming with lower energy consumption than digital beamforming. This approach would be worth exploring in future studies.

\section{Conclusions}

The upcoming 5G wireless technology is sparking numerous paradigm shifts in the mobile phone industry, since the use of the mm-Wave frequency band will alter antenna design and implementation requirements for modern smartphones. Currently, there is a knowledge gap in how to tackle the challenges imposed by $\mathrm{mm}$-Wave propagation in order to successfully integrate these new antennas onto a smartphone. Therefore, realistic models are necessary to predict the UE's behavior when facing the challenges of mm-Wave propagation before moving on to real life testing which, for such high frequencies, is an expensive and complex process.

The aim of the paper is to present a straightforward strategy that could be universally adopted to facilitate and influence the antenna design and collocation in the UE, taking into account the impact on the system performance at this stage, rather after a specific design is optimized taking into account only integration aspects. A two-step evaluation process is proposed, the mmWAESI method, to evaluate the coverage area potential and MIMO performance of different types of $\mathrm{mm}$-Wave antennas for implementation in a smartphone. This method facilitates mm-Wave antenna design and prototyping for smartphones by assessing the influence that array configuration, positioning in the smartphone, user hand grips, and variable environment scenarios have on the MIMO channel performance. Ultimately, it could also be used to test system level aspects such as different modulation schemes, coding, and channel estimation.

The PBS vs. MBS analysis was simply an example of the multiple functionalities of the mmWAESI method that showed how, despite the meager space available in the phone, mm-Wave arrays can still be added and bring the MIMO capacity enhancement to the mobile phone. Nevertheless, it is very clear that user blockage is a monumental challenge of mm-Wave communications, and results suggest that a multipanel beamforming solution should be integrated into the UE to improve coverage for all possible rotations and hand grips, using an appropriate switching scheme.

\section{Data Availability}

The data used to support the findings of this study are available from the corresponding author upon request.

\section{Conflicts of Interest}

The authors declare that they have no conflicts of interest.

\section{Acknowledgments}

This work was conducted at Intel Mobile Communications in Aalborg, Denmark, with the support of Instituto de Telecomunicações and Instituto Superior Técnico.

\section{References}

[1] J. G. Andrews, S. Buzzi, W. Choi et al., "What will 5G be?" IEEE Journal on Selected Areas in Communications, vol. 32, no. 6, pp. 1065-1082, 2014.

[2] Huawei, White Paper on "5G New Air Interface and Radio Access Virtualization", Huawei, Shenzhen, China, 2015.

[3] W. Hong, "Solving the 5G mobile antenna puzzle," IEEE Microwave Magazine, vol. 18, no. 7, pp. 86-102, 2017.

[4] W. Wonbin Hong, K. H. Kwang-Hyun Baek, Y. Youngju Lee, Y. Yoongeon Kim, and S. T. Seung-Tae Ko, "Study and prototyping of practically large-scale mmWave antenna systems for $5 \mathrm{G}$ cellular devices," IEEE Communications Magazine, vol. 52, no. 9, pp. 63-69, 2014.

[5] W. Hong, K.-H. Baek, and S. Ko, "Millimeter-wave 5G antennas for smartphones: overview and experimental demonstration," IEEE Transactions on Antennas and Propagation, vol. 65, no. 12, pp. 6250-6261, 2017.

[6] T. S. Rappaport, S. Sun, R. Mayzus et al., "Millimeter wave mobile communications for $5 \mathrm{G}$ cellular: it will work!" IEEE Access, vol. 1, pp. 335-349, 2013.

[7] W. Roh, J.-Y. Seol, J. Park et al., "Millimeter-wave beamforming as an enabling technology for $5 \mathrm{G}$ cellular communications: theoretical feasibility and prototype results," IEEE Communications Magazine, vol. 52, no. 2, pp. 106-113, 2014.

[8] F. Boccardi, R. W. Heath Jr, A. Lozano, T. L. Marzetta, and P. Popovski, "Five disruptive technology directions for $5 \mathrm{G}$," IEEE Communications Magazine, vol. 52, no. 2, pp. 74-80, 2014.

[9] W. Hong, K. Baek, Y. Lee, and Y. G. Kim, "Design and analysis of a low-profile $28 \mathrm{GHz}$ beam steering antenna solution for future 5G cellular applications," in Proceedings of the 2014 IEEE MTT-S International Microwave Symposium Digest, Tampa, FL, USA, June 2014.

[10] Y. Huo, X. Dong, and W. Xu, "5G cellular user equipment: from theory to practical hardware design," IEEE Access, vol. 5, pp. 13 992-14 010, 2017.

[11] O. Jo, J.-J. Kim, J. Yoon, D. Choi, and W. Hong, "Exploitation of dual-polarization diversity for $5 \mathrm{G}$ millimeter-wave MIMO beamforming systems," IEEE Transactions on Antennas and Propagation, vol. 65, no. 12, pp. 6646-6655, 2017.

[12] S. Sun, T. Rappaport, R. Heath Jr, A. Nix, and S. Rangan, "MIMO for millimeter-wave wireless communications: beamforming, spatial multiplexing, or both?" IEEE Communications Magazine, vol. 52, no. 12, pp. 110-121, 2014. 
[13] B. Yu, K. Yang, C.-Y.-D. Sim, and G. Yang, "A novel $28 \mathrm{GHz}$ beam steering array for $5 \mathrm{G}$ mobile device with metallic casing application," IEEE Transactions on Antennas and Propagation, vol. 66, no. 1, pp. 462-466, 2018.

[14] T. Wu, T. S. Rappaport, and C. M. Collins, "Safe for generations to come," IEEE Microwave Magazine, vol. 16, no. 2, pp. 65-84, 2015.

[15] K. Zhao, J. Helander, D. Sjöberg, S. He, T. Bolin, and Z. Ying, "User body effect on phased array in user equipment for the 5G mmWave communication system," IEEE Antennas and Wireless Propagation Letters, vol. 16, pp. 864-867, 2017.

[16] V. Raghavan, L. Akhoondzadeh-Asl, V. Podshivalov et al., "Statistical blockage modeling and robustness of beamforming in millimeter-wave systems," IEEE Transactions on Microwave Theory and Techniques, vol. 67, no. 7, pp. 30103024, 2019.

[17] M. Jensen and J. Wallace, "MIMO wireless channel modeling and experimental characterization," In Space-Time Processing for MIMO Communications, John Wiley \& Sons, Ltd, Hoboken, NJ, USA, 2005.

[18] ETSI, Study on Channel Model for Frequencies from 0.5 to 100 GHz (3GPP TR 38.901 Release 14), ETSI, Valbonne, France, 2018.

[19] CST Microwave Studio, "CST MWS workflow and solver overview," 2010.

[20] C. A. Balanis, Antenna Theory Analysis and Design, John Wiley \& Sons, Ltd, Hoboken, NJ, USA, 2005.

[21] Corning Inc, Corning Gorilla Glass 5 PI Sheet, Corning Inc, Corning, NY, USA, 2016.

[22] D. Andreuccettia and R. Fossi, "Dielectric properties of body tissues in the frequency range $10 \mathrm{~Hz}-100 \mathrm{GHz}$," 2021, http://niremf.ifac.cnr.it/tissprop/.

[23] Z. Ying, "Antennas in cellular phones for mobile communications," Proceedings of the IEEE, vol. 100, no. 72, pp. 2286-2296, 2012.

[24] J. Wang, Z. Lan, C. S. Sum et al., "Beamforming codebook design and performance evaluation for $60 \mathrm{GHz}$ wideband WPANs," in Proceedings of the 2009 IEEE Vehicular Technology Conference Fall, Anchorage, AK, USA, September 2009.

[25] S. Schindler and H. Mellein, Assessing a MIMO Channel, Rhode \& Schwarz, Munich, Germany, 2011.

[26] Keysight Technologies, MIMO Performance and Condition Number in LTE Test, Keysight Technologies, Santa Rosa, CA, USA, 2014.

[27] J. R. Costa, E. B. Lima, C. R. Medeiros, and C. A. Fernandes, "Evaluation of a new wideband slot array for MIMO performance enhancement in indoor WLANs," IEEE Transactions on Antennas and Propagation, vol. 59, no. 4, pp. 1200-1206, 2011. 